$4-1-2010$

\title{
Debye Series for Light Scattering by a Nonspherical Particle
}

Feng Xu

James A. Lock

Cleveland State University, j.lock@csuohio.edu

Gérard Gouesbet

Follow this and additional works at: https://engagedscholarship.csuohio.edu/sciphysics_facpub

Part of the Physics Commons

How does access to this work benefit you? Let us know!

Publisher's Statement

Copyright 2010 American Physical Society. Available on publisher's site at http://pra.aps.org/ abstract/PRA/v81/i4/e043824.

\section{Original Citation}

Xu, Feng, James A. Lock, and Gerard Gouesbet. "Debye Series for Light Scattering by a Nonspherical Particle." Physical Review A 81 (2010): 43824.

\section{Repository Citation}

Xu, Feng; Lock, James A.; and Gouesbet, Gérard, "Debye Series for Light Scattering by a Nonspherical Particle" (2010). Physics Faculty Publications. 10.

https://engagedscholarship.csuohio.edu/sciphysics_facpub/10

This Article is brought to you for free and open access by the Physics Department at EngagedScholarship@CSU. It has been accepted for inclusion in Physics Faculty Publications by an authorized administrator of EngagedScholarship@CSU. For more information, please contact library.es@csuohio.edu. 


\title{
Debye series for light scattering by a nonspherical particle
}

\author{
Feng Xu, ${ }^{1, *}$ James A. Lock, ${ }^{2}$ and Gérard Gouesbet ${ }^{3}$ \\ ${ }^{1}$ Fachgebiet Strömungslehre und Aerodynamik, Technische Universität Darmstadt, Petersenstraße 30, D-64289 Darmstadt, Germany \\ ${ }^{2}$ Department of Physics, Cleveland State University, Cleveland, Ohio 44115, USA \\ ${ }^{3}$ LESP/CORIA, CNRS, Université, et INSA de Rouen, F-76801 Rouen, France
}

(Received 9 January 2010; published 19 April 2010)

\begin{abstract}
The Debye series is developed for scattering of light by a homogeneous nonspherical particle to interpret the angular dependence of the scattered intensity in terms of various physical processes. In contrast to the previously developed Debye series for several regularly shaped particles that mirror the orthogonal curvilinear coordinate system where the variable-separation method can be applied, we develop and verify the Debye series in a coordinate-independent way using the extended boundary condition method. Verification computations are made for an oblate spheroidal water droplet of equivalent-volume sphere radius $10 \mu \mathrm{m}$.
\end{abstract}

DOI: 10.1103/PhysRevA.81.043824

PACS number(s): 42.25.Fx, 41.20.Jb, 42.68.Mj

\section{INTRODUCTION}

As an important tool for optical meteorological analysis and the theoretical basis for a number of elastic-scatteringbased particle characterization methods, Lorenz-Mie theory (LMT) [1] provides a rigorous way to describe scattering of a linearly polarized plane wave by a homogeneous sphere. To meet the requirements of more practical situations, LMT has been generalized in the past half century along two lines (i) beam shape and (ii) particle shape.

On one hand, since the advent of lasers, the development of LMT along line (i) received a vigorous effort. After some precursory work, such as in Refs. [2-4], a systematic study of laser-beam interaction with a class of regular particles has been contributed to by many researchers in the last three decades, as reviewed in Refs. [5,6]. Since the beam shape will differ for different purposes, the theory is developed under the name of generalized Lorenz-Mie theory (GLMT). GLMT provides a rigorous description of the transversely localized beam in terms of a set of beam shape coefficients (BSC's) and solves Maxwell equations subject to boundary conditions at the particle surface by the variable-separation method (VSM).

On the other hand, most particles existing in nature or produced in industrial processes are nonspherical. Therefore the development of LMT along line (ii) is significant for optical particle characterization by elastic-scattering techniques. Compared to the volume-based numerical solutions for light scattering by nonspherical particles which discritizes the entire volume, including the scatterer, into cells or substructures (e.g., the finite-difference time-domain method, the finite-element method, etc. [7]), the extended boundary condition method (EBCM, also known under the name of null-field method) introduced by Waterman is a surface-based solution [8]. It replaces the scattering object by a set of polarization currents over its surface. To analytically express the radiation problem, the Green dyadic is expanded in terms of vector spherical wave functions (VSWF's) and the scattered field coefficients are related to the incident field coefficients by a transition matrix ( $T$ matrix). But differing from the $T$-matrix formulation

*f3_xu@yahoo.com of LMT and GLMT where the VSM is used to evaluate the elements of the diagonal $T$ matrix [9], the EBCM evaluates the $T$-matrix elements by integrating vector spherical functions over the particle surface [10-12]. Moreover, the matrix is not diagonal as usually happens when particles are nonspherical.

Although the $T$-matrix formulation based on either VSM or EBCM can be used to numerically compute the scattered intensity, it cannot readily explain the physical mechanisms that cause various prominent features appearing in the intensity. In contrast, the Debye series formulation allows a detailed view of scattering by following the propagation of each partial wave (or equivalent light ray using van de Hulst's localization principle) inside the particle. By use of the Debye series, the far-zone scattered field can be decomposed into various orders with each order $p$ corresponding, in analogy to geometrical optics, to transmitted partial waves experiencing $(p-1)$ internal reflections, with $p=0$ corresponding to a purely reflected ray. This way, the physical cause of various features of the Mie scattering curve can be identified so that the scattering can be more clearly interpreted.

To date, the Debye series for plane-wave incidence was developed and verified for several types of particles. It is necessary to take a look back before introducing our work. The Debye series was first proposed by Debye in his studies on light scattering by a circular cylinder with normal plane-wave incidence [13]. The Debye series for the scattering amplitudes for plane-wave incidence on a sphere was first worked out by van der Pol and Bremmer [14] and was greatly popularized by Nussenzveig [15]. As a complement, the Debye series for the interior amplitudes for a sphere was derived in Ref. [16]. The Debye series for coated and multilayered spheres was derived in Ref. [17] and Refs. [18,19], respectively. The Debye series for a sphere with an embedded electric dipole source was derived in Ref. [20]. It is noteworthy that the Debye series with diagonal plane-wave incidence on a circular cylinder was derived in Ref. [21]. Generalizing the incident wave profile, the Debye series for shaped beam incidence was derived for a sphere through including a set of BSC's defined in GLMT [22]. A similar generalization was done afterward for a multilayered cylinder and for a multilayered sphere in Refs. [23,24], respectively. Recently, the Debye series for both plane-wave incidence and shaped-beam incidence on a homogeneous spheroid was worked out and verified [25]. 
However, all particle shapes involved above have mirrored a certain type of coordinate system (e.g., the spherical coordinate system, the cylindrical coordinate system, or the spheroidal coordinate system). This is a prerequisite for VSM to be applied so that by matching boundary conditions at the particle surface the coefficients for the expansion of the externally or internally reflected wave and transmitted waves can be determined in an analytical or numerical way. But the number of separable coordinate systems is very limited, namely, according to Kerker [26] and Morse and Feshbach [27], there are only 11 such separable systems for the wave equation.

The purpose of this article is to incorporate the Debye series decomposition into the EBCM for scattering by an arbitrarily shaped particle so as to have the strengths of both methods available in one formalism. It is our hope that the results derived here may be used to improve the interpretability of the various features of the scattered intensity when a plane wave is incident on an arbitrary shaped particle, a situation which commonly occurs in atmosphere measurement [28], or when an arbitrary-shaped laser beam is incident on an arbitrary-shaped particle, a situation which commonly occurs in two-phase flow and multiphase flow measurement [29].

The body of this study is organized as follows. In Sec. II A we briefly summarize the description of an arbitrary incident beam using the beam-shape coefficients of GLMT, and in Sec. II B we briefly summarize the EBCM formulation of light scattering by a particle of arbitrary shape. The verification of the Debye series for scattering of an electromagnetic wave by a particle of a given shape entails two separate calculations. The first is the determination of all the partial wave transmission and reflection amplitudes, and the second is the demonstration that when all the external and internal reflections are added together, the results exactly match the full partial wave scattering and interior amplitudes, with nothing left over and nothing missing. The partial wave transmission and reflection amplitudes are determined in Sec. III by extending the EBCM formulation from a radially incident standing wave to either a radially incoming traveling wave initially outside the particle or a radially outgoing traveling wave initially inside the particle. The results obtained in this section provide a practical way of computing any Debye series contribution to the partial wave scattering amplitudes. The progression of a single radially incoming partial wave as it successively internally reflects inside the particle is described in Sec. IV. At each interaction of the partial wave with the particle surface, transmitted and reflected contributions are produced in all partial wave channels for both transverse electric (TE) and transverse magnetic (TM) polarizations. Section IV serves as a transition between the computational point of view developed in Sec. III and the physical point of view required to complete the Debye series verification in the following section. In Sec. V the ideas concerning partial wave and polarization coupling introduced in Sec. IV are further developed. The terms of the Debye series are then added together and are shown to be identical to the full partial wave scattering and interior amplitudes, thus completing our verification of the Debye series for scattering of an arbitrary incident beam by a homogeneous dielectric particle of arbitrary shape. Verification computations using the EBCM Debye series developed in Sec. III are carried out in Sec. VI for an oblate spheroidal water droplet of equivalent-volume sphere radius $10 \mu \mathrm{m}$. The results of the scattering orders $p=0,1$, and 2 are analyzed by using Fraunhofer diffraction theory and geometrical optics, or compared with the computation by VSM Debye series in oblate spheroidal coordinates. Finally, in Sec. VII we state our conclusions. We also included two appendices. In Appendix A we determine the constant of proportionality between the vector spherical wave functions as used in GLMT calculations and those used in EBCM calculations. In Appendix B we formally sum the Debye series for both the interior and exterior partial wave amplitudes when they are considered as matrix equations in the partial wave and azimuthal mode numbers. We also develop a set of coupled recursion relations to determine the amplitude matrices to any order of scattering.

For readers primarily interested in Debye series EBCM numerical computations, the key equations are as follows. To calculate the scattered electric field in standard EBCM, one constructs the matrices $\boldsymbol{U}$ and $\boldsymbol{V}$ according to Eqs. (23) and (28), respectively. The partial wave scattering amplitudes are then obtained using Eq. (29). Finally, the scattered electric field is given by Eq. (9). The method for computing a particular Debye series component of the partial wave scattering amplitudes is similar. For external reflection, the matrices $\boldsymbol{U}^{D_{0}}$ and $\boldsymbol{V}^{D_{0}}$ are constructed according to Eqs. (42) and (48), respectively. The partial wave internal transmission and external reflection amplitudes $T^{21}$ and $R^{22}$ are obtained using Eqs. (41) and (49), respectively. Finally, the reflected electric field is given by Eq. (34). If one were instead interested in the contribution of transmission following a given number of internal reflections, the matrices $\boldsymbol{U}^{D_{p}}$ and $\boldsymbol{V}^{D_{p}}$ are constructed according to Eqs. (59) and (65), respectively. The partial wave external transmission and internal reflection amplitudes $T^{12}$ and $R^{11}$ are then obtained using Eqs. (58) and (66) where the incident amplitudes $I$ are defined following Eq. (51). Finally, the transmitted electric field is given by Eq. (54). These equations were used to obtain the results of Sec. VI.

\section{LIGHT SCATTERING THEORY}

Consider a monochromatic and arbitrarily oriented shaped beam that is incident on a nonspherical particle bounded by the surface $S$. We designate regions 2 and 1 to be the external and enclosed regions of the particle, respectively. We have the wave numbers $k_{2}\left(k_{2}=2 \pi / \lambda\right)$ and $k_{1}=m k_{2}$ for regions 2 and 1 , respectively, where $m$ is the complex refractive index of the particle relative to that of the medium. The particle and the medium are nonmagnetic, isotropic, and homogeneous, and the medium is infinite and nonabsorbing. For a nonmagnetic particle and medium, the permeabilities $\mu_{2}$ and $\mu_{1}$ are equal to the permeability of vacuum $\mu_{0}$.

The scattering problem is then considered to be the interaction of a particle with an arbitrary beam described by standing waves based on the spherical Bessel function of the first kind or with incoming or outgoing spherical multipole waves described by radially propagating waves based on the Hankel function of the second or first kind. GLMT and EBCM adopt the first way to deal with the incident wave, the spherical Bessel function of the first kind $j_{n}(k r)$ is used to generate the VSWF's of the first kind $\left(\mathbf{M}_{m n}^{(1)}, \mathbf{N}_{m n}^{(1)}\right)$, which are finite at 
the origin. The Debye series formulation, however, adopts the second way to deal with the incident wave, namely the spherical Hankel functions of the first kind $h_{n}^{(1)}$ and second kind $h_{n}^{(2)}$ are used to denote the spherically outgoing and incoming traveling waves, respectively. Accordingly, the VSWF's of the third and fourth kinds $\left(\mathbf{M}_{m n}^{(3)}, \mathbf{N}_{m n}^{(3)}\right)$ and $\left(\mathbf{M}_{m n}^{(4)}, \mathbf{N}_{m n}^{(4)}\right)$ are generated, respectively.

\section{A. GLMT description of the incident beam}

Assuming the time dependence $e^{-i \omega t}$, the beam can be described in spherical coordinates $(r, \theta, \varphi)$ associated with the particle's Cartesian coordinates $O X Y Z$ by a set of BSC's $G_{m n}^{\mathrm{TE}}$ and $G_{m n}^{\mathrm{TM}}$ in the following way

$$
\begin{gathered}
\mathbf{E}^{(i)}(\boldsymbol{r})=\sum_{n=1}^{\infty} \sum_{m=-n}^{+n}\left[G_{m n}^{\mathrm{TE}} \mathbf{M}_{m n}^{(1)}\left(k_{2} \boldsymbol{r}\right)+G_{m n}^{\mathrm{TM}} \mathbf{N}_{m n}^{(1)}\left(k_{2} \boldsymbol{r}\right)\right] \\
\mathbf{H}^{(i)}(\boldsymbol{r})=-i \frac{k_{2}}{\omega \mu_{2}} \sum_{n=1}^{\infty} \sum_{m=-n}^{+n}\left[G_{m n}^{\mathrm{TM}} \mathbf{M}_{m n}^{(1)}\left(k_{2} \boldsymbol{r}\right)+G_{m n}^{\mathrm{TE}} \mathbf{N}_{m n}^{(1)}\left(k_{2} \boldsymbol{r}\right)\right],
\end{gathered}
$$

where $\boldsymbol{r}$ is the position vector from the interior origin of coordinates $O$ to the field point (see Fig. 1).

Referring to the derivation in Appendix A, we can obtain the BSC's $\left(G_{m n}^{\mathrm{TE}}, G_{m n}^{\mathrm{TE}}\right)$ in Eqs. (1) and (2) in terms of the BSC's $\left(g_{m n}^{\mathrm{TE}}, g_{m n}^{\mathrm{TM}}\right)$ defined in the GLMT formulation $[9,30]$ by

$$
\begin{aligned}
G_{m n}^{\mathrm{TE}} & =\frac{k_{2}}{F_{m n}} \frac{(2 n+1) i^{n+1}}{n(n+1)} i \bar{g}_{-m n}^{\mathrm{TE}}, \\
G_{m n}^{\mathrm{TM}} & =\frac{k_{2}}{F_{m n}} \frac{(2 n+1) i^{n+1}}{n(n+1)} \bar{g}_{-m n}^{\mathrm{TM}},
\end{aligned}
$$

where the bar over $g$ denotes complex conjugation. The need for this new notation for the BSC's is caused by (1) the different assumptions of time dependence $\left(e^{-i \omega t}\right.$ in EBCM and $e^{i \omega t}$ in GLMT) and (2) the different definitions of VSWF's in GLMT as formulated by Gouesbet et al. [30] and the EBCM as formulated by Mishchenko et al. [31] (see Appendix A). The

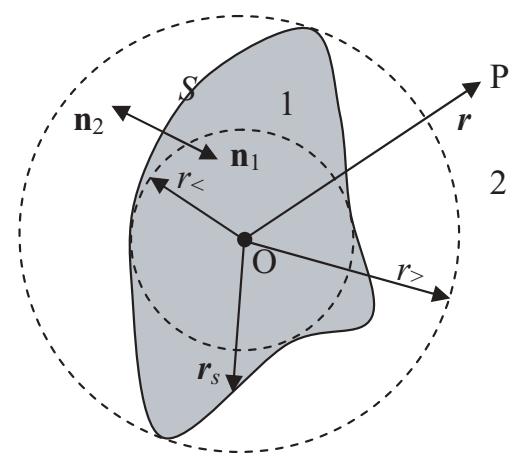

FIG. 1. Cross-section geometry of a homogeneous nonspherical particle bounded by the surface $S$. The radius $r_{>}$is that of the smallest circumscribing sphere centered on the origin $O$, and $r_{<}$is the radius of the largest concentric-inscribed sphere. The vector $\boldsymbol{r}$ goes from the origin to any point $P$ in space, and the vector $\boldsymbol{r}_{s}$ goes from the origin to any point on the particle surface. The vectors $\mathbf{n}_{2}$ and $\mathbf{n}_{1}$ are the outward and inward directed surface normals, respectively. constant of proportionality between the two sets of VSWF's is

$$
F_{m n}=\left\{\begin{array}{l}
{\left[\frac{(2 n+1)(n-m) !}{4 \pi n(n+1)(n+m) !}\right]^{1 / 2}, \quad m \geqslant 0} \\
(-1)^{m}\left[\frac{(2 n+1)(n+m) !}{4 \pi n(n+1)(n-m) !}\right]^{1 / 2}, \quad m<0 .
\end{array}\right.
$$

For a beam of arbitrary orientation in the particle coordinates, $g_{m n}^{\mathrm{TE}}$ and $g_{m n}^{\mathrm{TM}}$ can be rigorously evaluated, if the description of the incident beam used exactly satisfies Maxwell's equations, by a three-dimensional integral (or with a two-dimensional integral) of the radial components of the magnetic and the electric fields $H_{r}^{(i)}$ and $E_{r}^{(i)}$ in the spherical coordinates $(r, \theta, \varphi)[30,32,33]$

$$
\begin{aligned}
g_{m n}^{\mathrm{TE}}= & \frac{(2 n+1)^{2}}{2 \pi^{2} n(n+1) c_{n}^{\mathrm{pw}}} \frac{(n-|m|) !}{(n+|m|) !} \int_{0}^{\infty} r j_{n}\left(k_{2} r\right) \\
& \times \int_{0}^{2 \pi} \exp (-i m \phi) \int_{0}^{\pi} \frac{H_{r}^{(i)}(r, \theta, \phi)}{H_{0}} \\
& \times P_{|m| n}(\cos \theta) \sin \theta d \theta d \phi d\left(k_{2} r\right), \\
g_{m n}^{\mathrm{TM}}= & \frac{(2 n+1)^{2}}{2 \pi^{2} n(n+1) c_{n}^{\mathrm{pw}}} \frac{(n-|m|) !}{(n+|m|) !} \int_{0}^{\infty} r j_{n}\left(k_{2} r\right) \\
& \times \int_{0}^{2 \pi} \exp (-i m \phi) \int_{0}^{\pi} \frac{E_{r}^{(i)}(r, \theta, \phi)}{E_{0}} \\
& \times P_{|m| n}(\cos \theta) \sin \theta d \theta d \phi d\left(k_{2} r\right),
\end{aligned}
$$

where $P_{m n}$ is the associated Legendre function using Hobson's definition, and the partial wave amplitude of an incident plane wave $c_{n}^{\mathrm{pw}}$ is

$$
c_{n}^{\mathrm{pw}}=\frac{1}{k_{2}} i^{n-1}(-1)^{n} \frac{2 n+1}{n(n+1)} .
$$

To improve the computational efficiency of the BSC's, other methods were developed, namely the finite series method [34,35], the localized approximation or localized beam model approach ([36] and references therein), and the hybrid method combining the localized approximation and quadrature [37]. When off-axis and diagonal incidence of a beam is concerned, the translational addition theorem [38,39] provides an alternative way for BSC's computation.

Similarly, the electric component of the scattered field (denoted by the superscript " $s$ ") may be expanded in terms of the VSWF's of the third kind $\left(\mathbf{M}_{m n}^{(3)}, \mathbf{N}_{m n}^{(3)}\right)$

$$
\mathbf{E}^{(s)}(\boldsymbol{r})=\sum_{n=1}^{\infty} \sum_{m=-n}^{+n}\left[B_{m n} \mathbf{M}_{m n}^{(3)}\left(k_{2} \boldsymbol{r}\right)+A_{m n} \mathbf{N}_{m n}^{(3)}\left(k_{2} \boldsymbol{r}\right)\right] .
$$

Note that the definitions of $A_{m n}$ and $B_{m n}$ in Eq. (9) differ by a minus sign to what is standardly written down in Mie theory for $A_{m n}$ and $B_{m n}$ [40,41]. This new convention will have all of our final Debye series expansion of $A_{m n}$ and $B_{m n}$ differ from what is in the literature $[15,16]$ by a minus sign.

\section{B. EBCM formulation for light scattering by a particle}

Applying Schelkunoff's equivalence theorem to the scattered field $\left(\mathbf{E}^{(s)}, \mathbf{H}^{(s)}\right)$ in region 2 and to the negative of the incident field $\left(-\mathbf{E}^{(i)},-\mathbf{H}^{(i)}\right)$ in region 1 [42], a set of polarization currents $\mathbf{M}_{+}=\mathbf{E}_{+}\left(\boldsymbol{r}_{s}\right) \times \mathbf{n}_{2}=\left(\mathbf{E}^{(i)}+\mathbf{E}^{(s)}\right) \times \mathbf{n}_{2}$ and 
$\mathbf{J}_{+}=\mathbf{n}_{2} \times \mathbf{H}_{+}\left(\boldsymbol{r}_{s}\right)=\mathbf{n}_{2} \times\left(\mathbf{H}^{(i)}+\mathbf{H}^{(s)}\right)$ are generated where $\mathbf{n}_{2}$ is the outward directed surface normal and $\boldsymbol{r}_{s}$ is the position vector from the interior origin of coordinates $O$ to a point on the particle surface $S$. The polarization currents are distributed on $S$ and radiate the field $\left(\mathbf{E}^{(R)}(\boldsymbol{r}), \mathbf{H}^{(R)}(\boldsymbol{r})\right)$ into space. In region 2 , the radiation field is exactly the scattered field in the original problem, namely [10]

$$
\begin{aligned}
\mathbf{E}^{(R)}(\boldsymbol{r})= & \mathbf{E}^{(s)}(\boldsymbol{r})=\int_{S}\left\{i \omega \mu_{0}\left[\mathbf{n}_{2} \times \mathbf{H}_{+}\left(\boldsymbol{r}_{s}\right)\right] \cdot \overline{\bar{G}}\left(\boldsymbol{r}_{s} \mid \boldsymbol{r}\right)\right. \\
& \left.+\left[\mathbf{n}_{2} \times \mathbf{E}_{+}\left(\boldsymbol{r}_{s}\right)\right] \cdot\left[\nabla \times \overline{\bar{G}}\left(\boldsymbol{r}_{s} \mid \boldsymbol{r}\right)\right]\right\} d S
\end{aligned}
$$

where $\overline{\bar{G}}$ is the free space Green's dyadic

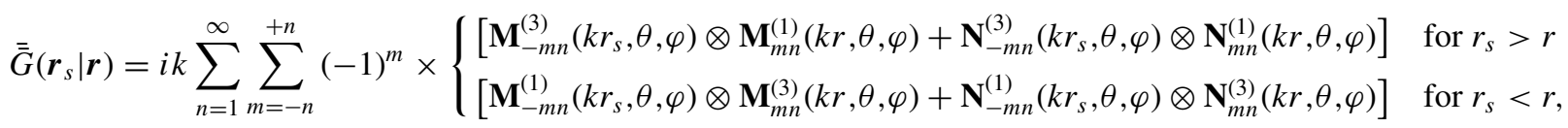

where $\otimes$ represents the dyadic product. In region 1 , the radiation field is the negative of the incident field

$$
\begin{aligned}
\mathbf{E}^{(R)}(\boldsymbol{r})= & -\mathbf{E}^{(i)}(\boldsymbol{r})=\int_{S}\left\{i \omega \mu_{0}\left[\mathbf{n}_{2} \times \mathbf{H}_{+}\left(\boldsymbol{r}_{s}\right)\right] \cdot \overline{\bar{G}}\left(\boldsymbol{r}_{s} \mid \boldsymbol{r}\right)\right. \\
& \left.+\left[\mathbf{n}_{2} \times \mathbf{E}_{+}\left(\boldsymbol{r}_{s}\right)\right] \cdot\left[\nabla \times \overline{\bar{G}}\left(\boldsymbol{r}_{s} \mid \boldsymbol{r}\right)\right]\right\} d S
\end{aligned}
$$

Denoting the radius of the smallest circumscribing sphere for the particle centered on the origin to be $r_{>}$(see Fig. 1), to ensure the convergence of the Green's function expansion of Eq. (11) we restrict $r$ to be $r>r_{>}$so that for $r_{s}$ we have $r>r_{s}$. The substitution of Eq. (10) to Eq. (9) gives

$$
\begin{aligned}
B_{m n}= & -(-1)^{m} k_{2} \int_{S}\left\{\omega \mu_{0}\left[\mathbf{n}_{2} \times \mathbf{H}_{+}\left(\boldsymbol{r}_{s}\right)\right] \cdot \mathbf{M}_{-m n}^{(1)}\left(k_{2} r_{s}, \theta, \varphi\right)\right. \\
& \left.-i k_{2}\left[\mathbf{n}_{2} \times \mathbf{E}_{+}\left(\boldsymbol{r}_{s}\right)\right] \cdot \mathbf{N}_{-m n}^{(1)}\left(k_{2} r_{s}, \theta, \varphi\right)\right\} d S, \\
A_{m n}= & -(-1)^{m} k_{2} \int_{S}\left\{\omega \mu_{0}\left[\mathbf{n}_{2} \times \mathbf{H}_{+}\left(\boldsymbol{r}_{s}\right)\right] \cdot \mathbf{N}_{-m n}^{(1)}\left(k_{2} r_{s}, \theta, \varphi\right)\right. \\
& \left.-i k_{2}\left[\mathbf{n}_{2} \times \mathbf{E}_{+}\left(\boldsymbol{r}_{s}\right)\right] \cdot \mathbf{M}_{-m n}^{(1)}\left(k_{2} r_{s}, \theta, \varphi\right)\right\} d S .
\end{aligned}
$$

Denoting the radius of the largest concentric inscribed sphere to be $r_{<}$(see Fig. 1), to ensure the convergence of the Green's function expansion of Eq. (11) we restrict $r$ to be $r<r_{<}$so that for all $r_{s}$ we have $r<r_{s}$. The substitution of Eq. (12) to Eq. (1) gives

$$
\begin{aligned}
G_{m n}^{\mathrm{TE}}= & (-1)^{m} k_{2} \int_{S}\left\{\omega \mu_{0}\left[\mathbf{n}_{2} \times \mathbf{H}_{+}\left(\boldsymbol{r}_{s}\right)\right] \cdot \mathbf{M}_{-m n}^{(3)}\left(k_{2} r_{s}, \theta, \varphi\right)\right. \\
& \left.-i k_{2}\left[\mathbf{n}_{2} \times \mathbf{E}_{+}\left(\boldsymbol{r}_{s}\right)\right] \cdot \mathbf{N}_{-m n}^{(3)}\left(k_{2} r_{s}, \theta, \varphi\right)\right\} d S \\
G_{m n}^{\mathrm{TM}}= & (-1)^{m} k_{2} \int_{S}\left\{\omega \mu_{0}\left[\mathbf{n}_{2} \times \mathbf{H}_{+}\left(\boldsymbol{r}_{s}\right)\right] \cdot \mathbf{N}_{-m n}^{(3)}\left(k_{2} r_{s}, \theta, \varphi\right)\right. \\
& \left.-i k_{2}\left[\mathbf{n}_{2} \times \mathbf{E}_{+}\left(\boldsymbol{r}_{s}\right)\right] \cdot \mathbf{M}_{-m n}^{(3)}\left(k_{2} r_{s}, \theta, \varphi\right)\right\} d S
\end{aligned}
$$

Continuity of the tangential components of the field $\left(\mathbf{E}_{+}, \mathbf{H}_{+}\right)$ at the particle surface $S$ is ensured by the boundary conditions

$$
\begin{aligned}
& \mathbf{n}_{2} \times \mathbf{E}_{+}\left(\boldsymbol{r}_{s}\right)=\mathbf{n}_{2} \times \mathbf{E}_{-}\left(\boldsymbol{r}_{s}\right), \\
& \mathbf{n}_{2} \times \mathbf{H}_{+}\left(\boldsymbol{r}_{s}\right)=\mathbf{n}_{2} \times \mathbf{H}_{-}\left(\boldsymbol{r}_{s}\right),
\end{aligned}
$$

where $\left(\boldsymbol{n} \times \mathbf{E}_{-}\right)$and $\left(\boldsymbol{n} \times \mathbf{H}_{-}\right)$are obtained from the interior field (denoted by the superscript "in") assumed to be expanded throughout region 1 in the following form

$$
\begin{gathered}
\mathbf{E}^{(\mathrm{in})}(\boldsymbol{r})=\sum_{n^{\prime}=1}^{\infty} \sum_{m^{\prime}=-n^{\prime}}^{+n^{\prime}}\left[D_{m^{\prime} n^{\prime}} \mathbf{M}_{m^{\prime} n^{\prime}}^{(1)}\left(k_{1} \boldsymbol{r}\right)+C_{m^{\prime} n^{\prime}} \mathbf{N}_{m^{\prime} n^{\prime}}^{(1)}\left(k_{1} \boldsymbol{r}\right)\right] \\
\mathbf{H}^{(\mathrm{in})}(\boldsymbol{r})=-i \frac{k_{1}}{\omega \mu_{0}} \sum_{n^{\prime}=1}^{\infty} \sum_{m^{\prime}=-n^{\prime}}^{+n^{\prime}}\left[D_{m^{\prime} n^{\prime}} \mathbf{N}_{m^{\prime} n^{\prime}}^{(1)}\left(k_{1} \boldsymbol{r}\right)\right. \\
\left.+C_{m^{\prime} n^{\prime}} \mathbf{M}_{m^{\prime} n^{\prime}}^{(1)}\left(k_{1} \boldsymbol{r}\right)\right] .
\end{gathered}
$$

Accordingly, we have

$$
\begin{aligned}
\mathbf{n}_{2} \times \mathbf{E}_{-}= & \sum_{n^{\prime}=1}^{\infty} \sum_{m^{\prime}=-n^{\prime}}^{+n^{\prime}}\left[D_{m^{\prime} n^{\prime}} \mathbf{n}_{2} \times \mathbf{M}_{m^{\prime} n^{\prime}}^{(1)}\left(k_{1} \boldsymbol{r}_{s}\right)\right. \\
& \left.+C_{m^{\prime} n^{\prime}} \mathbf{n}_{2} \times \mathbf{N}_{m^{\prime} n^{\prime}}^{(1)}\left(k_{1} \boldsymbol{r}_{s}\right)\right] \\
\mathbf{n}_{2} \times \mathbf{H}_{-}= & -i \frac{k_{1}}{\omega \mu_{0}} \sum_{n^{\prime}=1}^{\infty} \sum_{m^{\prime}=-n^{\prime}}^{+n^{\prime}}\left[D_{m^{\prime} n^{\prime}} \mathbf{n}_{2} \times \mathbf{N}_{m^{\prime} n^{\prime}}^{(1)}\left(k_{1} \boldsymbol{r}_{s}\right)\right. \\
& \left.+C_{m^{\prime} n^{\prime}} \mathbf{n}_{2} \times \mathbf{M}_{m^{\prime} n^{\prime}}^{(1)}\left(k_{1} \boldsymbol{r}_{s}\right)\right] .
\end{aligned}
$$

The substitution of Eqs. (17), (18), (21), and (22) into Eqs. (15) and (16) gives the following relation between the interior coefficients and the BSC's

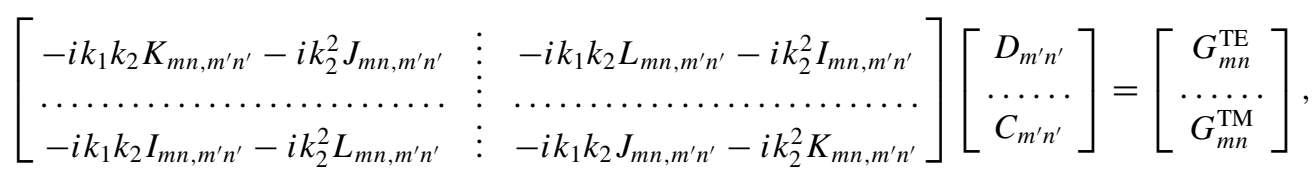


where

$$
\begin{aligned}
I_{m n, m^{\prime} n^{\prime}} & =(-1)^{m} \int_{S} \mathbf{n}_{2} \cdot \mathbf{M}_{m^{\prime} n^{\prime}}^{(1)}\left(k_{1} r_{s}, \theta, \varphi\right) \times \mathbf{M}_{-m n}^{(3)}\left(k_{2} r_{s}, \theta, \varphi\right) d S, \\
J_{m n, m^{\prime} n^{\prime}} & =(-1)^{m} \int_{S} \mathbf{n}_{2} \cdot \mathbf{M}_{m^{\prime} n^{\prime}}^{(1)}\left(k_{1} r_{s}, \theta, \varphi\right) \times \mathbf{N}_{-m n}^{(3)}\left(k_{2} r_{s}, \theta, \varphi\right) d S, \\
K_{m n, m^{\prime} n^{\prime}} & =(-1)^{m} \int_{S} \mathbf{n}_{2} \cdot \mathbf{N}_{m^{\prime} n^{\prime}}^{(1)}\left(k_{1} r_{s}, \theta, \varphi\right) \times \mathbf{M}_{-m n}^{(3)}\left(k_{2} r_{s}, \theta, \varphi\right) d S, \\
L_{m n, m^{\prime} n^{\prime}} & =(-1)^{m} \int_{S} \mathbf{n}_{2} \cdot \mathbf{N}_{m^{\prime} n^{\prime}}^{(1)}\left(k_{1} r_{s}, \theta, \varphi\right) \times \mathbf{N}_{-m n}^{(3)}\left(k_{2} r_{s}, \theta, \varphi\right) d S .
\end{aligned}
$$

If $C_{m n}$ and $D_{m n}$ were known, the substitution of Eqs. (17), (18), (21), and (22) into (13) and (14) gives the scattered field coefficients

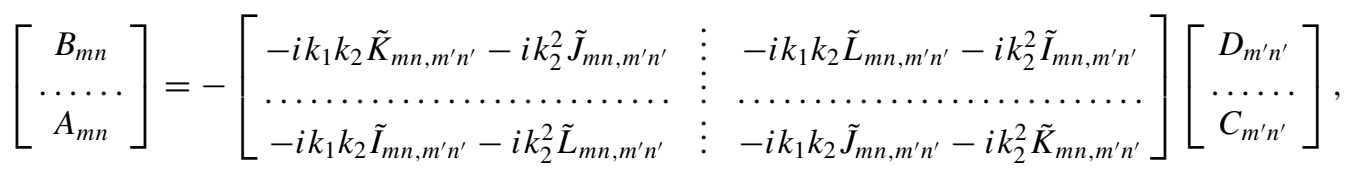

where $(\tilde{I}, \tilde{J}, \tilde{K}, \tilde{L})$ are similarly expressed as $(I, J, K, L)$ except that $\mathbf{M}_{-m n}^{(3)}$ is replaced by $\mathbf{M}_{-m n}^{(1)}$ and $\mathbf{N}_{-m n}^{(3)}$ is replaced by $\mathbf{N}_{-m n}^{(1)}$. If we designate the matrix in Eq. (23) as $\boldsymbol{U}$, the matrix in Eq. (28) as $\boldsymbol{V}$, and their multiplication $\boldsymbol{V} \boldsymbol{U}^{-1}$ as $\boldsymbol{T}$, Eq. (28) can be simplified to the $T$-matrix form as follows

$$
\left[\begin{array}{c}
B_{m n} \\
\cdots \cdots \\
A_{m n}
\end{array}\right]=-\boldsymbol{T}\left[\begin{array}{c}
G_{m^{\prime} n^{\prime}}^{\mathrm{TE}} \\
\cdots \cdots \\
G_{m^{\prime} n^{\prime}}^{\mathrm{TM}}
\end{array}\right]
$$

\section{DEBYE SERIES FOR A SPECIFIC ORDER $p$}

In Morse and Feshbach's derivation of the Green's dyadic expansion in terms of VSWF's [43], the Bessel function of first kind $j_{n}$ is used to denote the standing wave and the Hankel function of the first kind $h_{n}^{(1)}$ is used to denote the outgoing traveling wave. The Wronskian relation used by them to obtain
Eq. (11) is [44]

$$
j_{n}(k r) h_{n}^{(1)^{\prime}}(k r)-j_{n}^{\prime}(k r) h_{n}^{(1)}(k r)=\frac{i}{(k r)^{2}} .
$$

In the Debye series formulation, however, the spherical Hankel functions of the first kind $h_{n}^{(1)}$ and second kind $h_{n}^{(2)}$ are used to denote the outgoing and the incoming traveling waves, respectively. For external wave incidence on the particle surface $(p=0)$, the incident and transmitted waves are an incoming traveling wave and the externally reflected wave from the particle-medium surface is an outgoing traveling wave. For internal incidence at the particle surface $(p \geqslant 1)$, the incident and transmitted waves are an outgoing traveling wave and the internally reflected wave from the particle-medium surface is an incoming traveling wave. Whatever the case one has, the Wronskian relation becomes [44]

$$
h_{n}^{(2)}(k r) h_{n}^{(1)^{\prime}}(k r)-h_{n}^{(2)^{\prime}}(k r) h_{n}^{(1)}(k r)=\frac{2 i}{(k r)^{2}},
$$

and is used for the following expansion of the Green's dyadic for the Debye series indicated by the superscript " $D$ "

$$
\overline{\bar{G}}^{D}\left(\boldsymbol{r}_{s} \mid \boldsymbol{r}\right)=\frac{1}{2} i k \sum_{n=1}^{\infty} \sum_{m=-n}^{+n}(-1)^{m} \times \begin{cases}{\left[\mathbf{M}_{-m n}^{(3)}\left(k r_{s}, \theta, \varphi\right) \otimes \mathbf{M}_{m n}^{(4)}(k r, \theta, \varphi)+\mathbf{N}_{-m n}^{(3)}\left(k r_{s}, \theta, \varphi\right) \otimes \mathbf{N}_{m n}^{(4)}(k r, \theta, \varphi)\right]} & \text { for } r_{s}>r \\ {\left[\mathbf{M}_{-m n}^{(4)}\left(k r_{s}, \theta, \varphi\right) \otimes \mathbf{M}_{m n}^{(3)}(k r, \theta, \varphi)+\mathbf{N}_{-m n}^{(4)}\left(k r_{s}, \theta, \varphi\right) \otimes \mathbf{N}_{m n}^{(3)}(k r, \theta, \varphi)\right]} & \text { for } r_{s}<r .\end{cases}
$$

\section{A. External incidence at the particle surface, $p=0$}

Using the types of radial functions appropriate to the order $p=0$, the VSWF's $\left(\mathbf{M}_{m n}^{(4)}, \mathbf{N}_{m n}^{(4)}\right)$ and $\left(\mathbf{M}_{m n}^{(3)}, \mathbf{N}_{m n}^{(3)}\right)$ are generated for the description of the incident $(i)$ and externally reflected $(r)$ waves, respectively

$$
\mathbf{E}_{p=0}^{(i)}=\sum_{n_{0}=1}^{\infty} \sum_{m_{0}=-n_{0}}^{+n_{0}}\left[G_{m_{0} n_{0}}^{\mathrm{TE}} \mathbf{M}_{m_{0} n_{0}}^{(4)}\left(k_{2} \boldsymbol{r}\right)+G_{m_{0} n_{0}}^{\mathrm{TM}} \mathbf{N}_{m_{0} n_{0}}^{(4)}\left(k_{2} \boldsymbol{r}\right)\right]
$$

$$
\mathbf{E}_{p=0}^{(r)}=\sum_{n_{0}=1}^{\infty} \sum_{m_{0}=-n_{0}}^{+n_{0}}\left[R_{m_{0} n_{0}}^{22, \mathrm{TE}} \mathbf{M}_{m_{0} n_{0}}^{(3)}\left(k_{2} \boldsymbol{r}\right)+R_{m_{0} n_{0}}^{22, \mathrm{TM}} \mathbf{N}_{m_{0} n_{0}}^{(3)}\left(k_{2} \boldsymbol{r}\right)\right],
$$

where $R_{m_{0} n_{0}}^{22, \mathrm{TE}}$ and $R_{m_{0} n_{0}}^{22, \mathrm{TM}}$ are the external reflection amplitudes for the partial wave $\left(m_{0}, n_{0}\right)$ summed over contributions from all the incident partial wave channels. We then apply the equivalence theorem to the externally reflected and incident fields in our scattering problem. A set of reflected field sources $\mathbf{M}$ and $\mathbf{J}$ are assumed to be inside $S$ and radiate in all of 
space to produce $\left(\mathbf{E}^{(r)}, \mathbf{H}^{(r)}\right)$. When the field in region 2 is considered, the equivalence theorem states that $\mathbf{M}$ and $\mathbf{J}$ can be replaced by the equivalent surface currents $\mathbf{M}^{(r)}=\mathbf{E}^{(r)} \times \mathbf{n}_{2}$ and $\mathbf{J}^{(r)}=\mathbf{n}_{2} \times \mathbf{H}^{(r)}$, radiating the field $\left(\mathbf{E}^{(r)}, \mathbf{H}^{(r)}\right)$ outside $S$ and a null field inside $S$. Similarly, we consider another set of incident field sources $\mathbf{M}^{\prime}$ and $\mathbf{J}^{\prime}$ outside $S$, radiating in all of space to produce a field $\left(-\mathbf{E}^{(i)},-\mathbf{H}^{(i)}\right)$. Designating $\mathbf{n}_{1}$ as the inward directed unit surface normal, $\mathbf{M}^{\prime}$ and $\boldsymbol{J}^{\prime}$ can be replaced by the surface currents $\mathbf{M}^{(i)}=\left(-\mathbf{E}^{(i)}\right) \times \mathbf{n}_{1}$ and $\mathbf{J}_{i}=\mathbf{n}_{1} \times\left(-\mathbf{H}^{(i)}\right)$, radiating the field $\left(-\mathbf{E}^{(i)},-\mathbf{H}^{(i)}\right)$ inside $S$ and a null field outside $S$. Adding $\mathbf{M}^{(r)}$ to $\mathbf{M}^{(i)}$ and $\mathbf{J}^{(r)}$ to $\mathbf{J}^{(i)}$ produces the surface currents $\mathbf{M}_{+}=\left(\mathbf{E}^{(i)}+\mathbf{E}^{(r)}\right) \times \mathbf{n}_{2}=$ $\mathbf{E}_{+} \times \mathbf{n}_{2}$ and $\mathbf{J}_{+}=\mathbf{n}_{2} \times\left(\mathbf{H}^{(i)}+\mathbf{H}^{(r)}\right)=\mathbf{n}_{2} \times \mathbf{H}_{+}$, radiating $\left(-\mathbf{E}^{(i)},-\mathbf{H}^{(i)}\right)$ in region 1 and $\left(\mathbf{E}^{(r)}, \mathbf{H}^{(r)}\right)$ in region 2. A mathematical description of the radiation electric field $\mathbf{E}^{(R)}$ in this situation is

$$
\begin{array}{cc}
\mathbf{E}^{(R)}(\boldsymbol{r})=\mathbf{E}^{(r)}(\boldsymbol{r})=\int_{S}\left\{i \omega \mu_{0}\left[\mathbf{n}_{2} \times \mathbf{H}_{+}\left(\boldsymbol{r}_{s}\right)\right] \cdot \overline{\bar{G}}^{D}\left(\boldsymbol{r}_{s} \mid \boldsymbol{r}\right)+\left[\mathbf{n}_{2} \times \mathbf{E}_{+}\left(\boldsymbol{r}_{s}\right)\right] \cdot\left[\nabla \times \overline{\bar{G}}^{D}\left(\boldsymbol{r}_{s} \mid \boldsymbol{r}\right)\right]\right\} d S & \text { for } r_{s}<r, \\
\mathbf{E}^{(R)}(\boldsymbol{r})=-\mathbf{E}^{(i)}(\boldsymbol{r})=\int_{S}\left\{i \omega \mu_{0}\left[\mathbf{n}_{2} \times \mathbf{H}_{+}\left(\boldsymbol{r}_{s}\right)\right] \cdot \overline{\bar{G}}^{D}\left(\boldsymbol{r}_{s} \mid \boldsymbol{r}\right)+\left[\mathbf{n}_{2} \times \mathbf{E}_{+}\left(\boldsymbol{r}_{s}\right)\right] \cdot\left[\nabla \times \overline{\bar{G}}^{D}\left(\boldsymbol{r}_{s} \mid \boldsymbol{r}\right)\right]\right\} d S & \text { for } r_{s}>r .
\end{array}
$$

Adding the incident field to the radiation field produces a null field in region 1 and the external field $\left(\mathbf{E}^{(r)}+\mathbf{E}^{(i)}, \mathbf{H}^{(r)}+\mathbf{H}^{(i)}\right)$ in region 2 . The latter are exactly equivalent to the field in the original problem for $p=0$.

Demanding the continuity of the tangential components of the electric and magnetic fields on the surface $S,\left(\mathbf{n}_{2} \times \mathbf{E}_{+}\right)$ and $\left(\mathbf{n}_{2} \times \mathbf{H}_{+}\right)$can be obtained using Eqs. (17) and (18). Since the transmitted field denoted by the superscript $t$ is assumed to be expanded in terms of vector spherical waves in region 1

$$
\mathbf{E}_{p=0}^{(t)}=\sum_{n_{0}^{\prime}=1}^{\infty} \sum_{m_{0}^{\prime}=-n_{0}^{\prime}}^{+n_{0}^{\prime}}\left[T_{m_{0}^{\prime} n_{0}^{\prime}}^{21, \mathrm{TE}} \mathbf{M}_{m_{0}^{\prime} n_{0}^{\prime}}^{(4)}\left(k_{1} \boldsymbol{r}\right)+T_{m_{0}^{\prime} n_{0}^{\prime}}^{21, \mathrm{TM}} \mathbf{N}_{m_{0}^{\prime} n_{0}^{\prime}}^{(4)}\left(k_{1} \boldsymbol{r}\right)\right],
$$

$$
\begin{aligned}
\mathbf{H}_{p=0}^{(t)}= & -i \frac{k_{1}}{\omega \mu_{1}} \sum_{n_{0}^{\prime}=1}^{\infty} \sum_{m_{0}^{\prime}=-n_{0}^{\prime}}^{+n_{0}^{\prime}} \\
& \times\left[T_{m_{0}^{\prime} n_{0}^{\prime}}^{21, \mathrm{TM}} \mathbf{M}_{m_{0}^{\prime} n_{0}^{\prime}}^{(4)}\left(k_{1} \boldsymbol{r}\right)+T_{m_{0}^{\prime} n_{0}^{\prime}}^{21, \mathrm{TE}} \mathbf{N}_{m_{0}^{\prime} n_{0}^{\prime}}^{(4)}\left(k_{1} \boldsymbol{r}\right)\right]
\end{aligned}
$$

where the $T_{m_{0}^{\prime} n_{0}^{\prime}}^{21, \mathrm{TE}}$ and $T_{m_{0}^{\prime} n_{0}^{\prime}}^{21, \mathrm{TM}}$, differing from the bold $T$-matrix symbol $\boldsymbol{T}$ in Eq. (29), are the transmission amplitudes from region 2 to region 1 for the partial wave $\left(m_{0}^{\prime}, n_{0}^{\prime}\right)$ summed over contributions from all the incident partial wave channels, we have

$$
\begin{gathered}
\mathbf{n}_{2} \times \mathbf{E}_{+}=\mathbf{n}_{2} \times \mathbf{E}_{-}=\sum_{n_{0}^{\prime}=1}^{\infty} \sum_{m_{0}^{\prime}=-n_{0}^{\prime}}^{+n_{0}^{\prime}}\left[T_{m_{0}^{\prime} n_{0}^{\prime}}^{21, \mathrm{TE}} \mathbf{n}_{2} \times \mathbf{M}_{m_{0}^{\prime} n_{0}^{\prime}}^{(4)}\left(k_{1} \boldsymbol{r}_{s}\right)+T_{m_{0}^{\prime} n_{0}^{\prime}}^{21, \mathrm{TM}} \mathbf{n}_{2} \times \mathbf{N}_{m_{0}^{\prime} n_{0}^{\prime}}^{(4)}\left(k_{1} \boldsymbol{r}_{s}\right)\right], \\
\mathbf{n}_{2} \times \mathbf{H}_{+}=\mathbf{n}_{2} \times \mathbf{H}_{-}=-i \frac{k_{1}}{\omega \mu_{1}} \sum_{n_{0}^{\prime}=1}^{\infty} \sum_{m_{0}^{\prime}=-n_{0}^{\prime}}^{+n_{0}^{\prime}}\left[T_{m_{0}^{\prime} n_{0}^{\prime}}^{21, \mathrm{TE}} \mathbf{n}_{2} \times \mathbf{N}_{m_{0}^{\prime} n_{0}^{\prime}}^{(4)}\left(k_{1} \boldsymbol{r}_{s}\right)+T_{m_{0}^{\prime} n_{0}^{\prime}}^{21, \mathrm{TM}} \mathbf{n}_{2} \times \mathbf{M}_{m_{0}^{\prime} n_{0}^{\prime}}^{(4)}\left(k_{1} \boldsymbol{r}_{s}\right)\right] .
\end{gathered}
$$

Substituting Eqs. (32), (33), (39), and (40) into Eq. (36), after some algebra the transmitted-field coefficients are related to the incident-field coefficients by the matrix $\boldsymbol{U}^{D_{0}}$

$$
\boldsymbol{U}^{D_{0}}\left[\begin{array}{c}
T_{m_{0}^{\prime} n_{0}^{\prime}}^{21, \mathrm{TE}} \\
\cdots \cdots \\
T_{m_{0}^{\prime} n_{0}^{\prime}}^{21, \mathrm{TM}}
\end{array}\right]=\left[\begin{array}{c}
G_{m_{0} n_{0}}^{\mathrm{TE}} \\
\cdots \cdots \\
G_{m_{0} n_{0}}^{\mathrm{TM}}
\end{array}\right]
$$

where

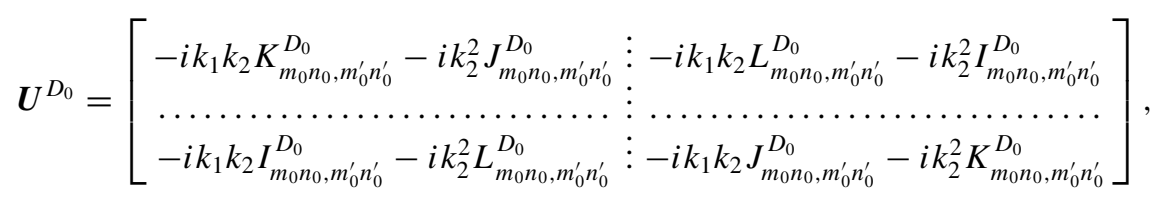

and the elements $\left(I^{D_{0}}, J^{D_{0}}, K^{D_{0}}, L^{D_{0}}\right)$ in the $\boldsymbol{U}^{D_{0}}$ matrix are evaluated by integrating vector spherical functions over the particle surface

$$
\begin{aligned}
& I_{m_{0} n_{0}, m_{0}^{\prime} n_{0}^{\prime}}^{D_{0}}=\frac{1}{2}(-1)^{m} \int_{S} \mathbf{n}_{2} \cdot \mathbf{M}_{m_{0}^{\prime} n_{0}^{\prime}}^{(4)}\left(k_{1} r_{s}, \theta, \varphi\right) \times \mathbf{M}_{-m_{0} n_{0}}^{(3)}\left(k_{2} r_{s}, \theta, \varphi\right) d S, \\
& J_{m_{0} n_{0}, m_{0}^{\prime} n_{0}^{\prime}}^{D_{0}}=\frac{1}{2}(-1)^{m} \int_{S} \mathbf{n}_{2} \cdot \mathbf{M}_{m_{0}^{\prime} n_{0}^{\prime}}^{(4)}\left(k_{1} r_{s}, \theta, \varphi\right) \times \mathbf{N}_{-m_{0} n_{0}}^{(3)}\left(k_{2} r_{s}, \theta, \varphi\right) d S, \\
& K_{m_{0} n_{0}, m_{0}^{\prime} n_{0}^{\prime}}^{D_{0}}=\frac{1}{2}(-1)^{m} \int_{S} \mathbf{n}_{2} \cdot \mathbf{N}_{m_{0}^{\prime} n_{0}^{\prime}}^{(4)}\left(k_{1} r_{s}, \theta, \varphi\right) \times \mathbf{M}_{-m_{0} n_{0}}^{(3)}\left(k_{2} r_{s}, \theta, \varphi\right) d S,
\end{aligned}
$$




$$
L_{m_{0} n_{0}, m_{0}^{\prime} n_{0}^{\prime}}^{D_{0}}=\frac{1}{2}(-1)^{m} \int_{S} \mathbf{n}_{2} \cdot \mathbf{N}_{m_{0}^{\prime} n_{0}^{\prime}}^{(4)}\left(k_{1} r_{s}, \theta, \varphi\right) \times \mathbf{N}_{-m_{0} n_{0}}^{(3)}\left(k_{2} r_{s}, \theta, \varphi\right) d S .
$$

Utilization of Eq. (34) instead of Eq. (33) and Eq. (35) instead of Eq. (36) gives the relation between the reflected-field coefficients and the transmitted-field coefficients

$$
\left[\begin{array}{l}
R_{m_{0} n_{0}}^{22, \mathrm{TE}} \\
\cdots \cdots \\
R_{m_{0} n_{0}}^{22, \mathrm{TM}}
\end{array}\right]=-\boldsymbol{V}^{D_{0}}\left[\begin{array}{l}
T_{m_{0}^{\prime} n_{0}^{\prime}}^{21, \mathrm{TE}} \\
\cdots \cdots \\
T_{m_{0}^{\prime} n_{0}^{\prime}}^{21, \mathrm{TM}}
\end{array}\right],
$$

where

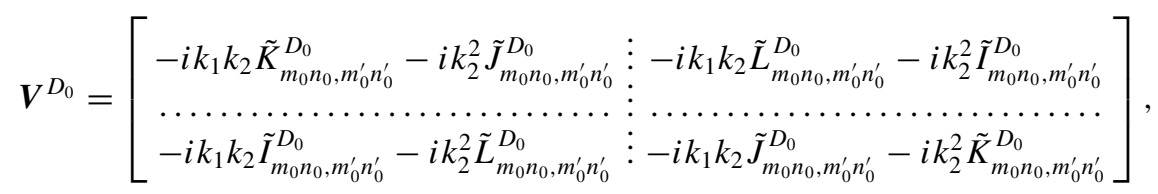

and the elements $\left(\tilde{I}^{D_{0}}, \tilde{J}^{D_{0}}, \tilde{K}^{D_{0}}, \tilde{L}^{D_{0}}\right)$ in the $\boldsymbol{V}^{D_{0}}$ matrix are similarly expressed as $\left(I^{D_{0}}, J^{D_{0}}, K^{D_{0}}, L^{D_{0}}\right)$ except that $\mathbf{M}_{-m_{0} n_{0}}^{(3)}$ is replaced by $\mathbf{M}_{-m_{0} n_{0}}^{(4)}$ and $\mathbf{N}_{-m_{0} n_{0}}^{(3)}$ is replaced by $\mathbf{N}_{-m_{0} n_{0}}^{(4)}$.

The substitution of Eq. (41) into Eq. (47) gives a direct $T$-matrix relation between the reflected-field coefficients and the incident-field coefficients for $p=0$, namely $\boldsymbol{T}^{D_{0}}=$ $\boldsymbol{V}^{D_{0}}\left[\boldsymbol{U}^{D_{0}}\right]^{-1}$ and

$$
\left[\begin{array}{l}
R_{m_{0} n_{0}}^{22, \mathrm{TE}} \\
\cdots \cdots \\
R_{m_{0} n_{0}}^{22, \mathrm{TM}}
\end{array}\right]=-\boldsymbol{T}^{D_{0}}\left[\begin{array}{l}
G_{m_{0}^{\prime} n_{0}^{\prime}}^{\mathrm{TE}} \\
\cdots \cdots \\
G_{m_{0}^{\prime} n_{0}^{\prime}}^{\mathrm{TM}}
\end{array}\right] .
$$

\section{B. Internal incidence at the particle surface, $p \geqslant 1$}

Using the types of radial functions appropriate to the orders $p \geqslant 1$, the VSWF's $\left(\mathbf{M}_{m n}^{(3)}, \mathbf{N}_{m n}^{(3)}\right)$ and $\left(\mathbf{M}_{m n}^{(4)}, \mathbf{N}_{m n}^{(4)}\right)$ are generated for the internally incident and reflected waves, respectively,

$$
\begin{aligned}
\mathbf{E}_{p}^{(i)} & =\sum_{n_{p}=1}^{\infty} \sum_{m_{p}=-n_{p}}^{+n_{p}}\left[I_{m_{p} n_{p}}^{\mathrm{TE}} \mathbf{M}_{m_{p} n_{p}}^{(3)}\left(k_{1} \boldsymbol{r}\right)+I_{m_{p} n_{p}}^{\mathrm{TM}} \mathbf{N}_{m_{p} n_{p}}^{(3)}\left(k_{1} \boldsymbol{r}\right)\right], \\
\mathbf{E}_{p}^{(r)} & =\sum_{n_{p}=1}^{\infty} \sum_{m_{p}=-n_{p}}^{+n_{p}}\left[R_{m_{p} n_{p}}^{11, \mathrm{TE}} \mathbf{M}_{m_{p} n_{p}}^{(4)}\left(k_{1} \boldsymbol{r}\right)+R_{m_{p} n_{p}}^{11, \mathrm{TM}} \mathbf{N}_{m_{p} n_{p}}^{(4)}\left(k_{1} \boldsymbol{r}\right)\right],
\end{aligned}
$$

where $R_{m_{p} n_{p}}^{11, \mathrm{TE}}$ and $R_{m_{p} n_{p}}^{11, \mathrm{TM}}$ are the internal reflection amplitudes for the partial wave $\left(m_{p}, n_{p}\right)$ summed over contributions from all the incident partial wave channels. Note that for the order $p=1$ the transmitted field of order $p=0$ is used as the incident field, namely $I_{m_{p} n_{p}}^{\mathrm{TE}}=T_{m_{p-1} n_{p-1}}^{21, \mathrm{TE}}$ and $I_{m_{p} n_{p}}^{\mathrm{TM}}=$ $T_{m_{p-1} n_{p-1}}^{21, \mathrm{TM}}$. For $p \geqslant 2$, the reflected field of order $(p-1)$ is used as the incident field, namely $I_{m_{p} n_{p}}^{\mathrm{TE}}=R_{m_{p-1} n_{p-1}}^{11, T E}$ and $I_{m_{p} n_{p}}^{\mathrm{TM}}=R_{m_{p-1} n_{p-1}}^{11, \mathrm{TM}}$. Again we apply the equivalence theorem to the fields for $p \geqslant 1$. Consider a set of reflected field sources $\mathbf{M}$ and $\mathbf{J}$ outside $S$, radiating in all of space to produce $\left(\mathbf{E}^{(r)}, \mathbf{H}^{(r)}\right)$. When the field in region 1 is considered, $\mathbf{M}$ and $\mathbf{J}$ can be replaced by the equivalent surface currents $\mathbf{M}^{(r)}=\mathbf{E}^{(r)} \times \mathbf{n}_{1}$ and $\mathbf{J}^{(r)}=\mathbf{n}_{1} \times \mathbf{H}^{(r)}$, radiating the field $\left(\mathbf{E}^{(r)}, \mathbf{H}^{(r)}\right)$ inside $S$ and a null field outside $S$. Consider another set of incident field sources $\mathbf{M}^{\prime}$ and $\mathbf{J}^{\prime}$ inside $S$, radiating in all of space to produce $\left(-\mathbf{E}^{(i)},-\mathbf{H}^{(i)}\right)$. When the field in region 2 is considered, $\mathbf{M}^{\prime}$ and $\mathbf{J}^{\prime}$ can be replaced by the surface currents $\mathbf{M}^{(i)}=\left(-\mathbf{E}^{(i)}\right) \times \mathbf{n}_{2}$ and $\mathbf{J}^{(i)}=\mathbf{n}_{2} \times\left(-\mathbf{H}^{(i)}\right)$, radiating the field $\left(-\mathbf{E}^{(i)},-\mathbf{H}^{(i)}\right)$ outside $S$ and a null field inside $S$. Adding $\mathbf{M}^{(r)}$ to $\mathbf{M}^{(i)}$ and $\mathbf{J}^{(r)}$ to $\mathbf{J}^{(i)}$ produces the surface currents $\mathbf{M}_{-}=\left(\mathbf{E}^{(i)}+\mathbf{E}^{(r)}\right) \times \mathbf{n}_{1}=\mathbf{E}_{-} \times \mathbf{n}_{1}$ and $\mathbf{J}_{-}=\mathbf{n}_{1} \times\left(\mathbf{H}^{(i)}+\right.$ $\left.\mathbf{H}^{(r)}\right)=\mathbf{n}_{1} \times \mathbf{H}_{-}$, radiating the field $\left(-\mathbf{E}^{(i)},-\mathbf{H}^{(i)}\right)$ outside $S$ (region 2) and the field $\left(\mathbf{E}^{(r)}, \mathbf{H}^{(r)}\right.$ ) inside $S$ (region 1). A mathematical description for the radiation electric field $\mathbf{E}^{(R)}$ becomes

$$
\begin{aligned}
\mathbf{E}^{(R)}(\boldsymbol{r})= & \mathbf{E}^{(r)}(\boldsymbol{r})=\int_{S}\left\{i \omega \mu_{0}\left[\mathbf{n}_{1} \times \mathbf{H}_{-}\left(\boldsymbol{r}_{s}\right)\right] \cdot \overline{\bar{G}}^{D}\left(\boldsymbol{r}_{s} \mid \boldsymbol{r}\right)\right. \\
& \left.+\left[\mathbf{n}_{1} \times \mathbf{E}_{-}\left(\boldsymbol{r}_{s}\right)\right] \cdot\left[\nabla \times \overline{\bar{G}}^{D}\left(\boldsymbol{r}_{s} \mid \boldsymbol{r}\right)\right]\right\} d S \text { for } r_{s}>r,
\end{aligned}
$$

$$
\begin{aligned}
\mathbf{E}^{(R)}(\boldsymbol{r})= & -\mathbf{E}^{(i)}(\boldsymbol{r})=\int_{S}\left\{i \omega \mu_{0}\left[\mathbf{n}_{1} \times \mathbf{H}_{-}\left(\boldsymbol{r}_{s}\right)\right] \cdot \overline{\bar{G}}^{D}\left(\boldsymbol{r}_{s} \mid \boldsymbol{r}\right)\right. \\
& \left.+\left[\mathbf{n}_{1} \times \mathbf{E}_{-}\left(\boldsymbol{r}_{s}\right)\right] \cdot\left[\nabla \times \overline{\bar{G}}^{D}\left(\boldsymbol{r}_{s} \mid \boldsymbol{r}\right)\right]\right\} d S \text { for } r_{s}<r .
\end{aligned}
$$

Adding the incident field to the radiation field produces a null field in region 2 and the internal field $\left(\mathbf{E}^{(r)}+\mathbf{E}^{(i)}, \mathbf{H}^{(r)}+\mathbf{H}^{(i)}\right)$ in region 1 . The latter is equivalent to the field in the original problem of $p \geqslant 1$.

Again demanding the continuity of the tangential components of the electric and magnetic fields across the surface $S$, $\left(\mathbf{n}_{1} \times \mathbf{E}_{-}\right)$and $\left(\mathbf{n}_{1} \times \mathbf{H}_{-}\right)$can be obtained using Eqs. (17) and (18) for the transmitted field, which is assumed to be expanded in terms of vector spherical waves in region 2

$$
\begin{aligned}
\mathbf{E}_{p}^{(t)}= & \sum_{n_{p}^{\prime}=1}^{\infty} \sum_{m_{p}^{\prime}=-n_{p}^{\prime}}^{+n_{p}^{\prime}}\left[T_{m_{p}^{\prime} n_{p}^{\prime}}^{12, \mathrm{TE}} \mathbf{M}_{m_{p}^{\prime} n_{p}^{\prime}}^{(3)}\left(k_{2} \boldsymbol{r}\right)+T_{m_{p}^{\prime} n_{p}^{\prime}}^{12, \mathrm{TM}} \mathbf{N}_{m_{p}^{\prime} n_{p}^{\prime}}^{(3)}\left(k_{2} \boldsymbol{r}\right)\right], \\
\mathbf{H}_{p}^{(t)}= & -i \frac{k_{2}}{\omega \mu_{2}} \sum_{n_{p}^{\prime}=1}^{\infty} \sum_{m_{p}^{\prime}=-n_{p}^{\prime}}^{+n_{p}^{\prime}}\left[T_{m_{p}^{\prime} n_{p}^{\prime}}^{12, \mathrm{TM}} \mathbf{M}_{m_{p}^{\prime} n_{p}^{\prime}}^{(3)}\left(k_{2} \boldsymbol{r}\right)\right. \\
& \left.+T_{m_{p}^{\prime} n_{p}^{\prime}}^{12, \mathrm{TE}} \mathbf{N}_{m_{p}^{\prime} n_{p}^{\prime}}^{(3)}\left(k_{2} \boldsymbol{r}\right)\right],
\end{aligned}
$$

where $T_{m_{p}^{\prime} n_{p}^{\prime}}^{12, \mathrm{TE}}$ and $T_{m_{p}^{\prime} n_{p}^{\prime}}^{12, \mathrm{TM}}$ are the transmission amplitudes from region 1 to region 2 for the partial wave $\left(m_{p}^{\prime}, n_{p}^{\prime}\right)$ summed 
over contributions from all the incident partial wave channels.

$$
\begin{aligned}
& \text { Accordingly, we have } \\
& \begin{aligned}
\mathbf{n}_{1} \times \mathbf{E}_{+}= & \mathbf{n}_{1} \times \mathbf{E}_{-}=\sum_{n_{p}^{\prime}=1}^{\infty} \sum_{m_{p}^{\prime}=-n_{p}^{\prime}}^{+n_{p}^{\prime}}\left[T_{m_{p}^{\prime} n_{p}^{\prime}}^{12, \mathrm{TE}} \mathbf{n}_{1} \times \mathbf{M}_{m_{p}^{\prime} n_{p}^{\prime}}^{(3)}\left(k_{2} \boldsymbol{r}_{s}\right)\right. \\
& \left.+T_{m_{p}^{\prime} n_{p}^{\prime}}^{12, \mathrm{TM}} \mathbf{n}_{1} \times \mathbf{N}_{m_{p}^{\prime} n_{p}^{\prime}}^{(3)}\left(k_{2} \boldsymbol{r}_{s}\right)\right] \\
\mathbf{n}_{1} \times \mathbf{H}_{+}= & \mathbf{n}_{1} \times \mathbf{H}_{-}=-i \frac{k_{2}}{\omega \mu_{2}} \sum_{n_{p}^{\prime}=1}^{\infty} \sum_{m_{p}^{\prime}=-n_{p}^{\prime}}\left[T_{m_{p}^{\prime} n_{p}^{\prime}}^{12, \mathrm{TE}} \mathbf{n}_{1}\right. \\
& \left.\times \mathbf{N}_{m_{p}^{\prime} n_{p}^{\prime}}^{(3)}\left(k_{2} \boldsymbol{r}_{s}\right)+T_{m_{p}^{\prime} n_{p}^{\prime}}^{12, \mathrm{TM}} \mathbf{n}_{1} \times \mathbf{M}_{m_{p}^{\prime} n_{p}^{\prime}}^{(3)}\left(k_{2} \boldsymbol{r}_{s}\right)\right]
\end{aligned}
\end{aligned}
$$

The substitution of Eqs. (32), (50), (56), and (57) into Eq. (53) gives the relation of the transmitted-field coefficients and the incident-field coefficients

$$
\boldsymbol{U}^{D_{p}}\left[\begin{array}{l}
T_{m_{p}^{\prime} n_{p}^{\prime}}^{12, \mathrm{TE}} \\
\cdots \cdots \\
T_{m_{p}^{\prime} n_{p}^{\prime}}^{12, \mathrm{TM}}
\end{array}\right]=\left[\begin{array}{l}
I_{m_{p} n_{p}}^{\mathrm{TE}} \\
\cdots \cdots \cdots \\
I_{m_{p} n_{p}}^{\mathrm{TM}}
\end{array}\right]
$$

where

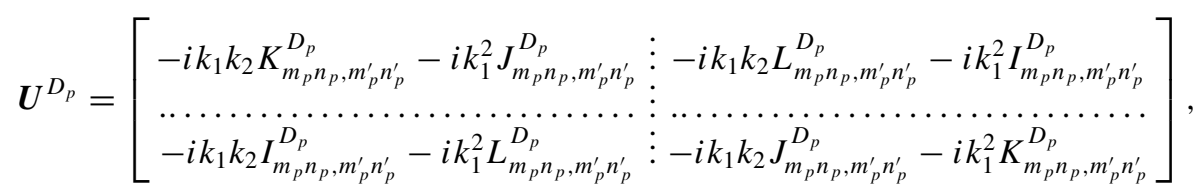

and the elements $\left(I^{D_{p}}, J^{D_{p}}, K^{D_{p}}, L^{D_{p}}\right)$ in the Debye-series based $\boldsymbol{U}^{D_{p}}$ matrix are evaluated by the following surface integrals

$$
\begin{aligned}
& I_{m_{p} n_{p}, m_{p}^{\prime} n_{p}^{\prime}}^{D_{p}}=\frac{1}{2}(-1)^{m} \int_{S} \mathbf{n}_{1} \cdot \mathbf{M}_{m_{p}^{\prime} n_{p}^{\prime}}^{(3)}\left(k_{2} r_{s}, \theta, \varphi\right) \mathbf{M}_{-m_{p}^{\prime} n_{p}^{\prime}}^{(4)}\left(k_{1} r_{s}, \theta, \varphi\right) d S, \\
& J_{m_{p} n_{p}, m_{p}^{\prime} n_{p}^{\prime}}^{D_{p}}=\frac{1}{2}(-1)^{m} \int_{S} \mathbf{n}_{1} \cdot \mathbf{M}_{m_{p}^{\prime} n_{p}^{\prime}}^{(3)}\left(k_{2} r_{s}, \theta, \varphi\right) \mathbf{N}_{-m_{p}^{\prime} n_{p}^{\prime}}^{(4)}\left(k_{1} r_{s}, \theta, \varphi\right) d S, \\
& K_{m_{p} n_{p}, m_{p}^{\prime} n_{p}^{\prime}}^{D_{p}}=\frac{1}{2}(-1)^{m} \int_{S} \mathbf{n}_{1} \cdot \mathbf{N}_{m_{p}^{\prime} n_{p}^{\prime}}^{(3)}\left(k_{2} r_{s}, \theta, \varphi\right) \mathbf{M}_{-m_{p}^{\prime} n_{p}^{\prime}}^{(4)}\left(k_{1} r_{s}, \theta, \varphi\right) d S, \\
& L_{m_{p} n_{p}, m_{p}^{\prime} n_{p}^{\prime}}^{D_{p}}=\frac{1}{2}(-1)^{m} \int_{S} \mathbf{n}_{1} \cdot \mathbf{N}_{m_{p}^{\prime} n_{p}^{\prime}}^{(3)}\left(k_{2} r_{S}, \theta, \varphi\right) \mathbf{N}_{-m_{p}^{\prime} n_{p}^{\prime}}^{(4)}\left(k_{1} r_{S}, \theta, \varphi\right) d S .
\end{aligned}
$$

The utilization of Eq. (50) instead of Eq. (51) and Eq. (52) instead of Eq. (53) gives the relation between the reflected-field coefficients and the transmitted-field coefficients

where

$$
\left[\begin{array}{l}
R_{m_{p} n_{p}}^{11, \mathrm{TE}} \\
\cdots \cdots \\
R_{m_{p} n_{p}}^{11, \mathrm{TM}}
\end{array}\right]=-\boldsymbol{V}^{D_{p}}\left[\begin{array}{c}
T_{m_{p}^{\prime} n_{p}^{\prime}}^{12 \mathrm{TE}} \\
\cdots \cdots \\
T_{m_{p}^{\prime} n_{p}^{\prime}}^{12, \mathrm{TM}}
\end{array}\right]
$$

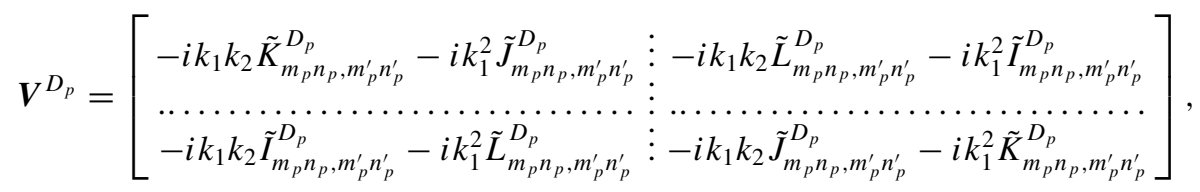

and the elements $\left(\tilde{I}^{D_{p}}, \tilde{J}^{D_{p}}, \tilde{K}^{D_{p}}, \tilde{L}^{D_{p}}\right)$ contained in the $\boldsymbol{V}^{D_{p}}$ matrix are similarly expressed as $\left(I^{D_{p}}, J^{D_{p}}, K^{D_{p}}, L^{D_{p}}\right)$ except that $\mathbf{M}_{-m_{p}^{\prime} n_{p}^{\prime}}^{(4)}$ is replaced by $\mathbf{M}_{-m_{p}^{\prime} n_{p}^{\prime}}^{(3)}$ and $\mathbf{N}_{-m_{p}^{\prime} n_{p}^{\prime}}^{(4)}$ is replaced by $\mathbf{N}_{-m_{p}^{\prime} n_{p}^{\prime}}^{(3)}$. The substitution of Eq. (58) into Eq. (64) gives a direct $T$-matrix relation between the reflected-field coefficients and the incident-field coefficients for $p \geqslant 1$, namely $\boldsymbol{T}^{D_{p}}=$ $\boldsymbol{V}^{D_{p}}\left[\boldsymbol{U}^{D_{p}}\right]^{-1}$ and

$$
\left[\begin{array}{l}
R_{m_{p} n_{p}}^{11, T E} \\
\cdots \cdots \\
R_{m_{p} n_{p}}^{11, T M}
\end{array}\right]=-\boldsymbol{T}^{D_{p}}\left[\begin{array}{l}
I_{m_{p}^{\prime} n_{p}^{\prime}}^{\mathrm{TE}} \\
\ldots \ldots \\
I_{m_{p}^{\prime} n_{p}^{\prime}}^{\mathrm{TM}}
\end{array}\right] .
$$

Note that for $p \geqslant 1$, the matrices $\boldsymbol{U}^{D_{p}}, \boldsymbol{V}^{D_{p}}$, and $\boldsymbol{T}^{D_{p}}$ are independent of $p$. Therefore they only need to be evaluated once. Moreover, the computation for axisymmetric particles can be much simplified since all the surface integrals vanish for $m_{p} \neq m_{p}^{\prime}$, which occurs for all $p \geqslant 0$.

With the known coefficients describing the incident field at the order $p$ obtained from the previous order $(p-$ 1 ), the transmitted and reflected fields at the order $p$ can be determined via Eqs. (58) and (66), respectively. In an iterative way, all orders of scattering are thus determined. 


\section{DEBYE SERIES FOR A SINGLE PARTIAL WAVE}

\section{A. External incidence at the particle surface, $p=0$}

In this section we examine the Debye series amplitudes from a more physically motivated point of view. We follow a single incident partial wave's propagation inside the particle, examining its successive interactions with the particle-medium interface and observing its evolution. For external incidence at the particle surface $(p=0)$, the incidence plane is formed by the beam propagation direction and the $z$ axis of the particle Cartesian coordinates. A single incident spherical partial wave with the electric field vibrating perpendicular to the incidence plane (i.e., TE polarization) is described by

$$
\begin{gathered}
\mathbf{E}_{p=0}^{(i), \mathrm{TE}}=G_{m_{0} n_{0}}^{\mathrm{TE}} \mathbf{M}_{m_{0} n_{0}}^{(4)}\left(k_{2} \boldsymbol{r}\right), \\
\mathbf{H}_{p=0}^{(i), \mathrm{TE}}=-\frac{i k_{2}}{\omega \mu_{2}}\left[G_{m_{0} n_{0}}^{\mathrm{TE}} \mathbf{N}_{m_{0} n_{0}}^{(4)}\left(k_{2} \boldsymbol{r}\right)\right]
\end{gathered}
$$

where $n_{0}$ is the partial wave number, $m_{0}$ is the azimuthal mode number, and $G_{m_{0} n_{0}}^{\mathrm{TE}}$ is the TE partial wave amplitude. Similarly, a single incident spherical partial wave with the magnetic field vibrating perpendicular to the incidence plane (i.e., TM polarization) is described by

$$
\begin{array}{r}
\mathbf{E}_{p=0}^{(i), \mathrm{TM}}=G_{m_{0} n_{0}}^{\mathrm{TM}} \mathbf{N}_{m_{0} n_{0}}^{(4)}\left(k_{2} \boldsymbol{r}\right), \\
\mathbf{H}_{p=0}^{(i), \mathrm{TM}}=-\frac{i k_{2}}{\omega \mu_{2}}\left[G_{m_{0} n_{0}}^{\mathrm{TM}} \mathbf{M}_{m_{0} n_{0}}^{(4)}\left(k_{2} \boldsymbol{r}\right)\right] .
\end{array}
$$

We define $R_{m_{1} n_{1}, m_{0} n_{0}}^{22}$ and $T_{m_{1} n_{1}, m_{0} n_{0}}^{21}$ as the portions reflected back into region 2 and transmitted into the particle, respectively, in the partial wave channel $\left(m_{1}, n_{1}\right)$ when the single partial wave $\left(m_{0}, n_{0}\right)$ of unit amplitude encounters the particle surface. Then the entire reflected and transmitted electric fields induced by a single TE incident partial wave $\left(m_{0}, n_{0}\right)$ with the amplitude $G_{m_{0} n_{0}}^{\mathrm{TE}}$ can be expressed as

$$
\begin{aligned}
\mathbf{E}_{p=0}^{(r)}= & \sum_{n_{1}=1}^{\infty} \sum_{m_{1}=-n_{1}}^{+n_{1}}\left[\left(R_{m_{1} n_{1}, m_{0} n_{0}}^{22, \mathrm{TE} / \mathrm{TE}} G_{m_{0} n_{0}}^{\mathrm{TE}}\right) \mathbf{M}_{m_{1} n_{1}}^{(3)}\left(k_{2} \boldsymbol{r}\right)\right. \\
& \left.+\left(R_{m_{1} n_{1}, m_{0} n_{0}}^{22, \mathrm{TM} / \mathrm{TE}} G_{m_{0} n_{0}}^{\mathrm{TE}}\right) \mathbf{N}_{m_{1} n_{1}}^{(3)}\left(k_{2} \boldsymbol{r}\right)\right]
\end{aligned}
$$

and

$$
\begin{aligned}
\mathbf{E}_{p=0}^{(t)}= & \sum_{n_{1}=1}^{\infty} \sum_{m_{1}=-n_{1}}^{+n_{1}}\left[\left(T_{m_{1} n_{1}, m_{0} n_{0}}^{21, \mathrm{TE} / \mathrm{TE}} G_{m_{0} n_{0}}^{\mathrm{TE}}\right) \mathbf{M}_{m_{1} n_{1}}^{(4)}\left(k_{1} \boldsymbol{r}\right)\right. \\
& \left.+\left(T_{m_{1} n_{1}, m_{0} n_{0}}^{21, \mathrm{TM} / \mathrm{TE}} G_{m_{0} n_{0}}^{\mathrm{TE}}\right) \mathbf{N}_{m_{1} n_{1}}^{(4)}\left(k_{1} \boldsymbol{r}\right)\right]
\end{aligned}
$$

respectively. The superscript TE/TE indicates the TE wave generated from the incident TE wave, and the superscript TM/TE indicates the TM wave generated from the incident TE wave. The situation considered here of a single incident TE partial wave represents a special case. The more general situation of both TE and TM incident partial waves is considered in Eqs. (75) and (94) through (101) when we relate these partial wave coupled transmission and reflection amplitudes to the entire partial wave amplitudes of Eqs. (34), (37), (51), and (54). Together with the TE wave generated from a TM incident wave, a cross polarization effect is induced at the order $p=0$. Multiplying Eqs. (71) and (72) by the factors $\left(-i k_{2} / \omega \mu_{2}\right)$ and $\left(-i k_{1} / \omega \mu_{1}\right)$, respectively, and interchanging
$\mathbf{M}_{m n}$ with $\mathbf{N}_{m n}$ gives the series describing the magnetic field. Note that the incident field in region 2 is radially incoming.

One can obtain analytical expressions for $R_{m_{1} n_{1}, m_{0} n_{0}}^{22}$ and $T_{m_{1} n_{1}, m_{0} n_{0}}^{21}$ for electromagnetic wave scattering by a sphere, coated sphere, cylinder, and scalar wave scattering by spheroid. But for electromagnetic wave scattering by more complicated nonspherical particles, we obtain the coefficients $R_{m_{1} n_{1}, m_{0} n_{0}}^{22}$ and $T_{m_{1} n_{1}, m_{0} n_{0}}^{21}$ numerically from the EBCM-based $T$-matrix solution to the reflected and the transmitted fields. For example, for the single incident TE partial wave $\left(m_{0}, n_{0}\right)$, the transmission amplitudes $T_{m_{1} n_{1}, m_{0} n_{0}}^{21, \mathrm{TE} / \mathrm{TE}}$ and $T_{m_{1} n_{1}, m_{0} n_{0}}^{21, \mathrm{TM} / \mathrm{TE}}$ are determined by assuming it has unit amplitude and neglecting other incident partial waves (namely for all partial waves $(m, n), G_{m n}^{\mathrm{TM}}=0$ and $G_{m n}^{\mathrm{TE}}=0$ except $G_{m n}^{\mathrm{TE}}=1$ as $m=m_{0}$ and $n=n_{0}$ ) so that viewing Eq. (41)

$$
\left[\begin{array}{c}
T_{m_{1} n_{1}, m_{0} n_{0}}^{21, \mathrm{TE} / \mathrm{TE}} \\
\cdots \cdots \cdots \\
T_{m_{1} n_{1}, m_{0} n_{0}}^{21, \mathrm{TM} / \mathrm{TE}}
\end{array}\right]=\left[\boldsymbol{U}^{D_{0}}\right]^{-1}\left[\begin{array}{c}
G_{m_{0} n_{0}}^{\mathrm{TE}}=1 \\
\cdots \cdots \cdots \\
0
\end{array}\right] .
$$

Similarly, viewing Eq. (49), $R_{m_{1} n_{1}, m_{0} n_{0}}^{22, \mathrm{TE} / \mathrm{TE}}$ and $R_{m_{1} n_{1}, m_{0} n_{0}}^{22, \mathrm{TM} / \mathrm{TE}}$ are determined by

$$
\left[\begin{array}{c}
R_{m_{1} n_{1}, m_{0} n_{0}}^{22, \mathrm{TE} / \mathrm{TE}} \\
\cdots \cdots \\
R_{m_{1} n_{1}, m_{0} n_{0}}^{22, \mathrm{TM} / \mathrm{TE}}
\end{array}\right]=-\boldsymbol{T}^{D_{0}}\left[\begin{array}{c}
G_{m_{0} n_{0}}^{\mathrm{TE}}=1 \\
\ldots \ldots \ldots . . \\
0
\end{array}\right] .
$$

Summing the contribution over all incident TE and TM partial waves, the externally reflected total electric field for the order $p=0$ becomes

$$
\begin{aligned}
\mathbf{E}_{p=0}^{(r)}= & \sum_{n_{1}=1}^{\infty} \sum_{m_{1}=-n_{1}}^{+n_{1}}\left[W_{m_{1} n_{1}}^{(r), \mathrm{TE}}(0) \mathbf{M}_{m_{1} n_{1}}^{(3)}\left(k_{2} \boldsymbol{r}\right)\right. \\
& \left.+W_{m_{1} n_{1}}^{(r), \mathrm{TM}}(0) \mathbf{N}_{m_{1} n_{1}}^{(3)}\left(k_{2} \boldsymbol{r}\right)\right],
\end{aligned}
$$

where

$$
\begin{aligned}
& {\left[\begin{array}{l}
W_{m_{1} n_{1}}^{(r), \mathrm{TE}}(0) \\
W_{m_{1} n_{1}}^{(r), \mathrm{TM}}(0)
\end{array}\right]} \\
& \quad=\sum_{n_{0}=1}^{\infty} \sum_{m_{0}=-n_{0}}^{+n_{0}}\left[\begin{array}{ll}
R_{m_{1} n_{1}, m_{0} n_{0}}^{22, \mathrm{TE}} & R_{m_{1} n_{1}, m_{0} n_{0}}^{22, \mathrm{TE} / \mathrm{TM}} \\
R_{m_{1} n_{1}, m_{0} n_{0}}^{22, \mathrm{TM}} & R_{m_{1} n_{1}, m_{0} n_{0}}^{22, \mathrm{TM}}
\end{array}\right]\left[\begin{array}{c}
G_{m_{0} n_{0}}^{\mathrm{TE}} \\
G_{m_{0} n_{0}}^{\mathrm{TM}}
\end{array}\right] .
\end{aligned}
$$

The total transmitted electric field inside the particle for the order $p=0$ becomes

$$
\begin{aligned}
\mathbf{E}_{p=0}^{(t)}= & \sum_{n_{1}=1}^{\infty} \sum_{m_{1}=-n_{1}}^{+n_{1}}\left[W_{m_{1} n_{1}}^{(t), \mathrm{TE}}(0) \mathbf{M}_{m_{1} n_{1}}^{(4)}\left(k_{1} \boldsymbol{r}\right)\right. \\
& \left.+W_{m_{1} n_{1}}^{(t), \mathrm{TM}}(0) \mathbf{N}_{m_{1} n_{1}}^{(4)}\left(k_{1} \boldsymbol{r}\right)\right],
\end{aligned}
$$

where

$$
\begin{aligned}
& {\left[\begin{array}{l}
W_{m_{1} n_{1}}^{(t), \mathrm{TE}}(0) \\
W_{m_{1} n_{1}}^{(t), \mathrm{TM}}(0)
\end{array}\right]} \\
& \quad=\sum_{n_{0}=1}^{\infty} \sum_{m_{0}=-n_{0}}^{+n_{0}}\left[\begin{array}{cc}
T_{m_{1} n_{1}, m_{0} n_{0}}^{21, \mathrm{TE} / \mathrm{TE}} & T_{m_{1} n_{1}, m_{0} n_{0}}^{21, \mathrm{TE} / \mathrm{TM}} \\
T_{m_{1} n_{1}, m_{0} n_{0}}^{21, \mathrm{TM}} & T_{m_{1} n_{1}, m_{0} n_{0}}^{21, \mathrm{TM}}
\end{array}\right]\left[\begin{array}{c}
G_{m_{0} n_{0}}^{\mathrm{TE}} \\
G_{m_{0} n_{0}}^{\mathrm{TM}}
\end{array}\right] .
\end{aligned}
$$




\section{B. Internal incidence at the particle surface, $p \geqslant 1$}

Assuming the linearity of the scattering process as expressed in Eqs. (94) through (101), the incident electric field for the wave-particle interaction for the second time $(p=1)$ is expressed in terms of the $p=0$ transmitted wave as

$$
\begin{aligned}
\mathbf{E}_{p=1}^{(i)}= & \sum_{n_{1}=1}^{\infty} \sum_{m_{1}=-n_{1}}^{+n_{1}}\left\{\left[\sum_{n_{0}=1}^{\infty} \sum_{m_{0}=-n_{0}}^{+n_{0}}\left(T_{m_{1} n_{1}, m_{0} n_{0}}^{21, \mathrm{TE} / \mathrm{TE}} G_{m_{0} n_{0}}^{\mathrm{TE}}+T_{m_{1} n_{1}, m_{0} n_{0}}^{21, \mathrm{TE} / \mathrm{TM}} G_{m_{0} n_{0}}^{\mathrm{TM}}\right)\right] \mathbf{M}_{m_{1} n_{1}}^{(3)}\left(k_{1} \boldsymbol{r}\right)\right. \\
& \left.+\left[\sum_{n_{0}=1}^{\infty} \sum_{m_{0}=-n_{0}}^{+n_{0}}\left(T_{m_{1} n_{1}, m_{0} n_{0}}^{21, \mathrm{TM} / \mathrm{TE}} G_{m_{0} n_{0}}^{\mathrm{TE}}+T_{m_{1} n_{1}, m_{0} n_{0}}^{21, \mathrm{TM}} G_{m_{0} n_{0}}^{\mathrm{TM}}\right)\right] \mathbf{N}_{m_{1} n_{1}}^{(3)}\left(k_{1} \boldsymbol{r}\right)\right\}
\end{aligned}
$$

Taking into account the amplitudes of all incident TE and TM partial waves of a shaped beam as done in Eqs. (75) and (77), the reflected and transmitted electric fields of order $p=1$ are

$$
\begin{aligned}
\mathbf{E}_{p=1}^{(r)}= & \sum_{n_{2}=1}^{\infty} \sum_{m_{2}=-n_{2}}^{+n_{2}}\left(\left\{\sum _ { n _ { 1 } = 1 } ^ { \infty } \sum _ { m _ { 1 } = - n _ { 1 } } ^ { + n _ { 1 } } \sum _ { n _ { 0 } = 1 } ^ { \infty } \sum _ { m _ { 0 } = - n _ { 0 } } ^ { + n _ { 0 } } \left[R_{m_{2} n_{2}, m_{1} n_{1}}^{11, \mathrm{TE} / \mathrm{TE}}\left(T_{m_{1} n_{1}, m_{0} n_{0}}^{21, \mathrm{TE} / \mathrm{TE}} G_{m_{0} n_{0}}^{\mathrm{TE}}+T_{m_{1} n_{1}, m_{0} n_{0}}^{21, \mathrm{TE} / \mathrm{TM}} G_{m_{0} n_{0}}^{\mathrm{TM}}\right)+R_{m_{2} n_{2}, m_{1} n_{1}}^{11, \mathrm{TE} / \mathrm{TM}}\left(T_{m_{1} n_{1}, m_{0} n_{0}}^{21, \mathrm{TM} / \mathrm{TE}} G_{m_{0} n_{0}}^{\mathrm{TE}}\right.\right.\right.\right. \\
& \left.\left.\left.+T_{m_{1} n_{1}, m_{0} n_{0}}^{21, \mathrm{TM} / \mathrm{TM}} G_{m_{0} n_{0}}^{\mathrm{TM}}\right)\right]\right\} \mathbf{M}_{m_{2} n_{2}}^{(4)}\left(k_{1} \boldsymbol{r}\right) \\
& +\left\{\sum _ { n _ { 1 } = 1 } ^ { \infty } \sum _ { m _ { 1 } = - n _ { 1 } } ^ { + n _ { 1 } } \sum _ { n _ { 0 } = 1 } ^ { \infty } \sum _ { m _ { 0 } = - n _ { 0 } } ^ { + n _ { 0 } } \left[R_{m_{2} n_{2}, m_{1} n_{1}}^{11, \mathrm{TM} / \mathrm{TE}}\left(T_{m_{1} n_{1}, m_{0} n_{0}}^{21, \mathrm{TE} / \mathrm{TE}} G_{m_{0} n_{0}}^{\mathrm{TE}}+T_{m_{1} n_{1}, m_{0} n_{0}}^{21, \mathrm{TE} / \mathrm{TM}} G_{m_{0} n_{0}}^{\mathrm{TM}}\right)+R_{m_{2} n_{2}, m_{1} n_{1}}^{11, \mathrm{TM} / \mathrm{TM}}\left(T_{m_{1} n_{1}, m_{0} n_{0}}^{21, \mathrm{TM} / \mathrm{TE}} G_{m_{0} n_{0}}^{\mathrm{TE}}\right.\right.\right. \\
& \left.\left.\left.\left.+T_{m_{1} n_{1}, m_{0} n_{0}}^{21, \mathrm{TM} / \mathrm{TM}} G_{m_{0} n_{0}}^{\mathrm{TM}}\right)\right]\right\} \mathbf{N}_{m_{2} n_{2}}^{(4)}\left(k_{1} \boldsymbol{r}\right)\right),
\end{aligned}
$$

and

$$
\begin{aligned}
\mathbf{E}_{p=1}^{(t)}= & \sum_{n_{2}=1}^{\infty} \sum_{m_{2}=-n_{2}}^{+n_{2}}\left(\left\{\sum _ { n _ { 1 } = 1 } ^ { \infty } \sum _ { m _ { 1 } = - n _ { 1 } } ^ { + n _ { 1 } } \sum _ { n _ { 0 } = 1 } ^ { \infty } \sum _ { m _ { 0 } = - n _ { 0 } } ^ { + n _ { 0 } } \left[T_{m_{2} n_{2}, m_{1} n_{1}}^{12, \mathrm{TE} / \mathrm{TE}}\left(T_{m_{1} n_{1}, m_{0} n_{0}}^{21, \mathrm{TE} / \mathrm{TE}} G_{m_{0} n_{0}}^{\mathrm{TE}}+T_{m_{1} n_{1}, m_{0} n_{0}}^{21, \mathrm{TE} / \mathrm{TM}} G_{m_{0} n_{0}}^{\mathrm{TM}}\right)+T_{m_{2} n_{2}, m_{1} n_{1}}^{12, \mathrm{TE} / \mathrm{TM}}\left(T_{m_{1} n_{1}, m_{0} n_{0}}^{21, \mathrm{TM} / \mathrm{TE}} G_{m_{0} n_{0}}^{\mathrm{TE}}\right.\right.\right.\right. \\
& \left.\left.\left.+T_{m_{1} n_{1}, m_{0} n_{0}}^{21, \mathrm{TM} / \mathrm{TM}} G_{m_{0} n_{0}}^{\mathrm{TM}}\right)\right]\right\} \mathbf{M}_{m_{2} n_{2}}^{(3)}\left(k_{2} \boldsymbol{r}\right) \\
& +\left\{\sum _ { n _ { 1 } = 1 } ^ { \infty } \sum _ { m _ { 1 } = - n _ { 1 } } ^ { + n _ { 1 } } \sum _ { n _ { 0 } = 1 } ^ { \infty } \sum _ { m _ { 0 } = - n _ { 0 } } ^ { + n _ { 0 } } \left[T_{m_{2} n_{2}, m_{1} n_{1}}^{12, \mathrm{TM} / \mathrm{TE}}\left(T_{m_{1} n_{1}, m_{0} n_{0}}^{21, \mathrm{TE} / \mathrm{TE}} G_{m_{0} n_{0}}^{\mathrm{TE}}+T_{m_{1} n_{1}, m_{0} n_{0}}^{21, \mathrm{TE} / \mathrm{TM}} G_{m_{0} n_{0}}^{\mathrm{TM}}\right)+T_{m_{2} n_{2}, m_{1} n_{1}}^{12, \mathrm{TM} / \mathrm{TM}}\left(T_{m_{1} n_{1}, m_{0} n_{0}}^{21, \mathrm{TM} / \mathrm{TE}} G_{m_{0} n_{0}}^{\mathrm{TE}}\right.\right.\right. \\
& \left.\left.\left.\left.+T_{m_{1} n_{1}, m_{0} n_{0}}^{21, \mathrm{TM} / \mathrm{TM}} G_{m_{0} n_{0}}^{\mathrm{TM}}\right)\right]\right\} \mathbf{N}_{m_{2} n_{2}}^{(4)}\left(k_{2} \boldsymbol{r}\right)\right)
\end{aligned}
$$

respectively, where the reflected and transmitted portions of the partial wave $\left(m_{2}, n_{2}\right)$ coupled to the incident partial wave $\left(m_{1}, n_{1}\right)$ of unit amplitude are designated as $R_{m_{2} n_{2}, m_{1} n_{1}}^{11}$ and $T_{m_{2} n_{2}, m_{1} n_{1}}^{12}$, respectively, for the order $p=1$.

In contrast to order $p=0$, the incident field is now radially outgoing in region 1 . But as was the case for the order $p=0$, one cannot get an explicit expression for $R_{m_{2} n_{2}, m_{1} n_{1}}^{11}$ and $T_{m_{2} n_{2}, m_{1} n_{1}}^{12}$ for electromagnetic wave scattering by a nonspherical particle. Therefore again these amplitudes are determined from the EBCM-based $T$-matrix solutions to the reflected and transmitted fields. For example, for the single TE partial wave $\left(m_{2}, n_{2}\right)$, the transmission coefficients $T_{m_{2} n_{2}, m_{1} n_{1}}^{12, \mathrm{TE} / \mathrm{TE}}$ and $T_{m_{2} n_{2}, m_{1} n_{1}}^{12, \mathrm{TM}}$ coupled to the incident partial wave $\left(m_{1}, n_{1}\right)$ are determined by assuming its amplitude to be unity and neglecting other incident partial waves so that viewing Eq. (58)

$$
\left[\begin{array}{c}
T_{m_{2} n_{2}, m_{1} n_{1}}^{12, \mathrm{TE} / \mathrm{TE}} \\
\cdots \cdots \cdots \\
T_{m_{2} n_{2}, m_{1} n_{1}}^{12, \mathrm{TM} / \mathrm{TE}}
\end{array}\right]=\left[\boldsymbol{U}^{D_{p}}\right]^{-1}\left[\begin{array}{c}
I_{m_{1} n_{1}}^{\mathrm{TE}}=1 \\
\cdots \cdots \cdots \\
0
\end{array}\right],
$$

and viewing Eq. (66)

$$
\left[\begin{array}{c}
R_{m_{2} n_{2}, m_{1} n_{1}}^{11, \mathrm{TE} / \mathrm{TE}} \\
\cdots \cdots \cdots \\
R_{m_{2} n_{2}, m_{1} n_{1}}^{11, \mathrm{TM} / \mathrm{TE}}
\end{array}\right]=-\boldsymbol{T}^{D_{p}}\left[\begin{array}{c}
I_{m_{1} n_{1}}^{\mathrm{TE}}=1 \\
\cdots \cdots \cdots \\
0
\end{array}\right] .
$$


Without loss of generality, the incident electric field inside the particle for an arbitrary order $p(p \geqslant 1)$ can be described in the following way

$$
\mathbf{E}_{p}^{(i)}=\sum_{n_{p}=1}^{\infty} \sum_{m_{p}=-n_{p}}^{+n_{p}}\left[W_{m_{p} n_{p}}^{(i), \mathrm{TE}}(p) \mathbf{M}_{m_{p} n_{p}}^{(3)}\left(k_{1} \boldsymbol{r}\right)+W_{m_{p} n_{p}}^{(i), \mathrm{TM}}(p) \mathbf{N}_{m_{p} n_{p}}^{(3)}\left(k_{1} \boldsymbol{r}\right)\right]
$$

where $W_{m_{p} n_{p}}^{(i), \mathrm{TE}}$ and $W_{m_{p} n_{p}}^{(i), \mathrm{TM}}$ are given by

$$
\left[\begin{array}{l}
W_{m_{p} n_{p}}^{(i), \mathrm{TE}}(p) \\
W_{m_{p} n_{p}}^{(i), \mathrm{TM}}(p)
\end{array}\right]=\sum_{n_{p-1}=1}^{\infty} \sum_{m_{p-1}=-n_{p-1}}^{n_{p-1}} \cdots \sum_{n_{0}=1}^{\infty} \sum_{m_{0}=-n_{0}}^{+n_{0}}\left[\begin{array}{ll}
F_{m_{p} n_{p}, m_{p-1} n_{p-1}}^{\mathrm{TE} / \mathrm{TE}} & F_{m_{p} n_{p}, m_{p-1} n_{p-1}}^{\mathrm{TE} / \mathrm{TM}} \\
F_{m_{p} n_{p}, m_{p-1} n_{p-1}}^{\mathrm{TM} / \mathrm{TE}} & F_{m_{p} n_{p}, m_{p-1} n_{p-1}}^{\mathrm{TM} / \mathrm{TM}}
\end{array}\right] \cdots\left[\begin{array}{l}
F_{m_{1} n_{1}, m_{0} n_{0}}^{\mathrm{TE} / \mathrm{TE}} F_{m_{1} n_{1}, m_{0} n_{0}}^{\mathrm{TE} / \mathrm{TM}} \\
F_{m_{1} n_{1}, m_{0} n_{0}}^{\mathrm{TM} / \mathrm{TE}} F_{m_{1} n_{1}, m_{0} n_{0}}^{\mathrm{TM} / \mathrm{TM}}
\end{array}\right]\left[\begin{array}{c}
G_{m_{0} n_{0}}^{\mathrm{TE}} \\
G_{m_{0} n_{0}}^{\mathrm{TM}}
\end{array}\right],
$$

where $F=T^{21}$ for $n_{i}$ with $i=1$ and $F=R^{11}$ for $n_{i}$ with $i \geqslant 2$.

The full reflected electric field inside the particle at the order $p$ can be described by taking the incident field [Eq. (84)] and determining its reflected portion in region 1 so that

$$
\begin{aligned}
\mathbf{E}_{p}^{(r)}= & \sum_{n_{p+1}=1}^{\infty} \sum_{m_{p+1}=-n_{p+1}}^{+n_{p+1}}\left[W_{m_{p+1} n_{p+1}}^{(r), \mathrm{TE}}(p) \mathbf{M}_{m_{p+1} n_{p+1}}^{(4)}\left(k_{1} \boldsymbol{r}\right)\right. \\
& \left.+W_{m_{p+1} n_{p+1}}^{(r), \mathrm{TM}}(p) \mathbf{N}_{m_{p+1} n_{p+1}}^{(4)}\left(k_{1} \boldsymbol{r}\right)\right]
\end{aligned}
$$

where

$$
\begin{aligned}
& {\left[\begin{array}{l}
W_{m_{p+1} n_{p+1}}^{(r), \mathrm{TE}}(p) \\
W_{m_{p+1} n_{p+1}}^{(r), \mathrm{TM}}(p)
\end{array}\right]} \\
& =\sum_{n_{p}=1}^{\infty} \sum_{m_{p}=-n_{p}}^{+n_{p}}\left[\begin{array}{ll}
R_{m_{p+1} n_{p+1}, m_{p} n_{p}}^{11, \mathrm{TE} / \mathrm{TE}} & R_{m_{p+1} n_{p+1}, m_{p} n_{p}}^{11, \mathrm{TE} / \mathrm{TM}} \\
R_{m_{p+1} n_{p+1}, m_{p} n_{p}}^{11, \mathrm{TM} / \mathrm{TE}} & R_{m_{p+1} n_{p+1}, m_{p} n_{p}}^{11, \mathrm{TM} / \mathrm{TM}}
\end{array}\right] \\
& \quad \times\left[\begin{array}{c}
W_{m_{p} n_{p}}^{(i), \mathrm{TE}}(p) \\
W_{m_{p} n_{p}}^{(i), \mathrm{TM}}(p)
\end{array}\right] .
\end{aligned}
$$

The electric field transmitted out of the particle is obtained by taking the incident field and determining its transmitted portion in region 2 so that

$$
\begin{aligned}
\mathbf{E}_{p}^{(t)}= & \sum_{n_{p+1}=1}^{\infty} \sum_{m_{p+1}=-n_{p+1}}^{+n_{p+1}}\left[W_{m_{p+1} n_{p+1}}^{(t), \mathrm{TE}}(p) \mathbf{M}_{m_{p+1} n_{p+1}}^{(3)}\left(k_{2} \boldsymbol{r}\right)\right. \\
& \left.+W_{m_{p+1} n_{p+1}}^{(t), \mathrm{TM}}(p) \mathbf{N}_{m_{p+1} n_{p+1}}^{(3)}\left(k_{2} \boldsymbol{r}\right)\right],
\end{aligned}
$$

where

$$
\begin{aligned}
& {\left[\begin{array}{l}
W_{m_{p+1} n_{p+1}}^{(t), \mathrm{TE}}(p) \\
W_{m_{p+1} n_{p+1}}^{(t), \mathrm{TM}}(p)
\end{array}\right]} \\
& =\sum_{n_{p}=1}^{\infty} \sum_{m_{p}=-n_{p}}^{+n_{p}}\left[\begin{array}{cc}
T_{m_{p+1} n_{p+1}, m_{p} n_{p}}^{12, \mathrm{TE} / \mathrm{TE}} & T_{m_{p+1} n_{p+1}, m_{p} n_{p}}^{12, \mathrm{TE} / \mathrm{TM}} \\
T_{m_{p+1} n_{p+1}, m_{p} n_{p}}^{12, \mathrm{TM} / \mathrm{TE}} & T_{m_{p+1} n_{p+1}, m_{p} n_{p}}^{12, \mathrm{TM} / \mathrm{TM}}
\end{array}\right] \\
& \quad \times\left[\begin{array}{c}
W_{m_{p} n_{p}}^{(i), \mathrm{TE}}(p) \\
W_{m_{p} n_{p}}^{(i), \mathrm{TM}}(p)
\end{array}\right] .
\end{aligned}
$$

In each order of scattering the TE/TM cross-polarization part contains the term $F_{m_{p+1} n_{p+1}, m_{p} n_{p}}^{\mathrm{TE} / \mathrm{TM}}$ in $W^{\mathrm{TE}}$ and the TM/TE cross-polarization part contains the term $F_{m_{p+1} n_{p+1}, m_{p} n_{p}}^{\mathrm{TM} / \mathrm{TE}}$ in $W^{\mathrm{TM}}$, where $F=R^{11}$ for Eq. (87) and $F=T^{12}$ for Eq. (89).
We have not yet addressed the question of whether when all such terms have been added together, the result is exactly equal to the full partial wave amplitudes of the beam being scattered by the particle. This question is taken up in the next section. In addition, it is noteworthy that the expansion of the scattered field in the form of Eq. (9) and the expansion of the reflected field in the form of Eqs. (34), (71), and (75) are numerically valid outside the circumscribed sphere, and the expansion of the reflected field in the form of Eqs. (51), (80), and (86) is valid inside the inscribed sphere. Generally they cannot be applied for accurate field calculation in the near-zone region due to the nonconvergence of the Green dyadic expansion. The near-zone region is the domain bounded by the particle's surface and the circumscribed sphere in the Mie scattering problem and in the Debye series problem of the order $p=0$. In the Debye series problem of orders $p \geqslant 1$, it is the domain bounded by the particle's surface and the inscribed sphere. To calculate the field in the near-zone region in the Mie scattering problem, Doicu and Wriedt [45] proposed to use the integral representation of the scattered field, namely Eq. (10). Their method might also be applied in the Debye series problem through using the integral representation of the reflected field, namely Eq. (35) for the order $p=0$ and Eq. (52) for the orders $p \geqslant 1$, with the surface currents determined from the boundary conditions Eqs. (39) and (40) for $p=0$ and Eqs. (56) and (57) for $p \geqslant 1$.

\section{VERIFICATION OF DEBYE SERIES}

The verification of the Debye series decomposition of the various partial wave amplitudes for scattering of an incident electromagnetic wave by a particle of general shape differs from that for scattering by a sphere or a circular cylinder at normal incidence because all the amplitudes involved are elements of matrices rather than being scalars. This occurs because scattering by a particle of arbitrary shape couples different partial waves, different azimuthal modes, and different polarizations between the incident and scattered states. The verification of the Debye series consists of five steps, the first three of which were already addressed in Secs. II through IV.

Step 1: Consider the full Mie scattering problem of a shaped electromagnetic wave incident on the particle. The incident and scattered electric fields are given by Eqs. (1) and (9), respectively, and the interior electric field is given by Eq. (19). In these equations the coefficients $A_{m n}$ and $B_{m n}$ are the partial wave scattering amplitudes and $C_{m n}$ and $D_{m n}$ 
are the partial wave interior amplitudes corresponding to the incident wave field strengths $G_{m n}^{\mathrm{TE}}$ and $G_{m n}^{\mathrm{TM}}$. Linearity of the scattering process requires that each of the interior and scattering amplitudes depends linearly on the field strength of each of the incident partial waves $\left(m_{1}, n_{1}\right)$ as

$$
\begin{aligned}
A_{m n} & =\sum_{n_{1}=1}^{\infty} \sum_{m_{1}=-n_{1}}^{+n_{1}}\left[A_{m n, m_{1} n_{1}}^{\mathrm{TM} / \mathrm{TE}} G_{m_{1} n_{1}}^{\mathrm{TE}}+A_{m n, m_{1} n_{1}}^{\mathrm{TM} / \mathrm{TM}} G_{m_{1} n_{1}}^{\mathrm{TM}}\right], \\
B_{m n} & =\sum_{n_{1}=1}^{\infty} \sum_{m_{1}=-n_{1}}^{+n_{1}}\left[B_{m n, m_{1} n_{1}}^{\mathrm{TE} / \mathrm{TE}} G_{m_{1} n_{1}}^{\mathrm{TE}}+B_{m n, m_{1} n_{1}}^{\mathrm{TE} / \mathrm{TM}} G_{m_{1} n_{1}}^{\mathrm{TM}}\right], \\
C_{m n}= & \sum_{n_{1}=1}^{\infty} \sum_{m_{1}=-n_{1}}^{+n_{1}}\left[C_{m n, m_{1} n_{1}}^{\mathrm{TM} / \mathrm{TE}} G_{m_{1} n_{1}}^{\mathrm{TE}}+C_{m n, m_{1} n_{1}}^{\mathrm{TM} / \mathrm{TM}} G_{m_{1} n_{1}}^{\mathrm{TM}}\right], \\
D_{m n}= & \sum_{n_{1}=1}^{\infty} \sum_{m_{1}=-n_{1}}^{+n_{1}}\left[D_{m n, m_{1} n_{1}}^{\mathrm{TE} / \mathrm{TE}} G_{m_{1} n_{1}}^{\mathrm{TE}}+D_{m n, m_{1} n_{1}}^{\mathrm{TE} / \mathrm{TM}} G_{m_{1} n_{1}}^{\mathrm{TM}}\right],
\end{aligned}
$$

and each polarization of each incoming partial wave $\left(m_{1}, n_{1}\right)$ contributes to both polarizations of all of the scattered and interior partial waves $(m, n)$. For any given scattering geometry the numerical values of the partial wave scattering and interior amplitudes may be determined using the EBCM of Sec. II B.

Step 2: Consider a radially incoming wave with partial wave coefficients $(1 / 2) G_{m n}^{\mathrm{TE}}$ and $(1 / 2) G_{m n}^{\mathrm{TM}}$ incident on the particle. The interaction of the radially incident wave with the particle surface creates both a radially outgoing reflected wave in the external medium with partial wave amplitudes $R_{m n}^{22}$ for each polarization and a radially incoming transmitted wave inside the particle with partial amplitudes $T_{m n}^{21}$ for each polarization. The incident, reflected, and interior electric fields for this case are given by Eqs. (33), (34), and (37), respectively.

Again linearity of the scattering process dictates that each partial wave reflection and transmission amplitude depends on the strength of all of the incoming partial waves as

$$
R_{m n}^{22, \mathrm{TE}}=\frac{1}{2} \sum_{n_{1}=1}^{\infty} \sum_{m_{1}=-n_{1}}^{+n_{1}}\left[R_{m n, m_{1} n_{1}}^{22, \mathrm{TE} / \mathrm{TE}} G_{m_{1} n_{1}}^{\mathrm{TE}}+R_{m n, m_{1} n_{1}}^{22, \mathrm{TE} / \mathrm{TM}} G_{m_{1} n_{1}}^{\mathrm{TM}}\right]
$$

$R_{m n}^{22, \mathrm{TM}}=\frac{1}{2} \sum_{n_{1}=1}^{\infty} \sum_{m_{1}=-n_{1}}^{+n_{1}}\left[R_{m n, m_{1} n_{1}}^{22, \mathrm{TM} / \mathrm{TE}} G_{m_{1} n_{1}}^{\mathrm{TE}}+R_{m n, m_{1} n_{1}}^{22, \mathrm{TM} / \mathrm{TM}} G_{m_{1} n_{1}}^{\mathrm{TM}}\right]$,

$T_{m n}^{21, \mathrm{TE}}=\frac{1}{2} \sum_{n_{1}=1}^{\infty} \sum_{m_{1}=-n_{1}}^{+n_{1}}\left[T_{m n, m_{1} n_{1}}^{21, \mathrm{TE} / \mathrm{TE}} G_{m_{1} n_{1}}^{\mathrm{TE}}+T_{m n, m_{1} n_{1}}^{21, \mathrm{TE} / \mathrm{TM}} G_{m_{1} n_{1}}^{\mathrm{TM}}\right]$,

$T_{m n}^{21, \mathrm{TM}}=\frac{1}{2} \sum_{n_{1}=1}^{\infty} \sum_{m_{1}=-n_{1}}^{+n_{1}}\left[T_{m n, m_{1} n_{1}}^{21, \mathrm{TM} / \mathrm{TE}} G_{m_{1} n_{1}}^{\mathrm{TE}}+T_{m n, m_{1} n_{1}}^{21, \mathrm{TM}} G_{m_{1} n_{1}}^{\mathrm{TM}}\right]$

This linearity property was assumed in the development of Sec. IV A when a single partial wave was incident on the particle surface [see Eqs. (71) and (72)]. For any given scattering geometry the numerical value of all these amplitudes may be determined using the EBCM for the Debye series of Secs. III A and IV A.
Step 3: Lastly, consider a radially outgoing wave inside the particle with partial wave coefficients $(1 / 2) I_{m n}^{\mathrm{TE}}$ and $(1 / 2) I_{m n}^{\mathrm{TM}}$ that is incident on the particle surface. The interaction of the incident wave with the particle surface creates both a radially outgoing transmitted wave in the external medium with partial wave amplitudes $T_{m n}^{12}$ for each polarization and a radially incoming reflected wave inside the particle with partial amplitudes $R_{m n}^{11}$ for each polarization. The incident, reflected, and interior electric fields for this case are given by Eqs. (50), (51), and (54), respectively. Again each of the partial wave reflection and transmission amplitudes depends on the strength of all of the incoming partial waves by

$$
\begin{aligned}
R_{m n}^{11, \mathrm{TE}} & =\frac{1}{2} \sum_{n_{1}=1}^{\infty} \sum_{m_{1}=-n_{1}}^{+n_{1}}\left[R_{m n, m_{1} n_{1}}^{11, \mathrm{TE} / \mathrm{TE}} I_{m_{1} n_{1}}^{\mathrm{TE}}+R_{m n, m_{1} n_{1}}^{11, \mathrm{TE} / \mathrm{TM}} I_{m_{1} n_{1}}^{\mathrm{TM}}\right], \\
R_{m n}^{11, \mathrm{TM}} & =\frac{1}{2} \sum_{n_{1}=1}^{\infty} \sum_{m_{1}=-n_{1}}^{+n_{1}}\left[R_{m n, m_{1} n_{1}}^{11, \mathrm{TM} / \mathrm{TE}} I_{m_{1} n_{1}}^{\mathrm{TE}}+R_{m n, m_{1} n_{1}}^{11, \mathrm{TM} / \mathrm{TM}} I_{m_{1} n_{1}}^{\mathrm{TM}}\right], \\
T_{m n}^{12, \mathrm{TE}} & =\frac{1}{2} \sum_{n_{1}=1}^{\infty} \sum_{m_{1}=-n_{1}}^{+n_{1}}\left[T_{m n, m_{1} n_{1}}^{12, \mathrm{TE} / \mathrm{TE}} I_{m_{1} n_{1}}^{\mathrm{TE}}+T_{m n, m_{1} n_{1}}^{12, \mathrm{TM}} I_{m_{1} n_{1}}^{\mathrm{TM}}\right], \\
T_{m n}^{12, \mathrm{TM}} & =\frac{1}{2} \sum_{n_{1}=1}^{\infty} \sum_{m_{1}=-n_{1}}^{+n_{1}}\left[T_{m n, m_{1} n_{1}}^{12, \mathrm{TM} / \mathrm{TE}} I_{m_{1} n_{1}}^{\mathrm{TE}}+T_{m n, m_{1} n_{1}}^{12, \mathrm{TM} / \mathrm{TM}} I_{m_{1} n_{1}}^{\mathrm{TM}}\right] .
\end{aligned}
$$

The numerical values of all these amplitudes may be determined using the EBCM for Debye series of Secs. III B and IV B.

Step 4: To evaluate the interior amplitudes $C_{m n}$ and $D_{m n}$ for the full scattering problem of step 1 in terms of the partial wave reflection and transmission amplitudes, the interior field of Eq. (37) of step 2 is added to the total interior field of Eq. (50) plus Eq. (51) of step 3. The incident beam coefficients $I_{m_{1} n_{1}}^{\mathrm{TE}}$ and $I_{m_{1} n_{1}}^{\mathrm{TM}}$ are then varied while $G_{m_{1} n_{1}}^{\mathrm{TE}}$ and $G_{m_{1} n_{1}}^{\mathrm{TM}}$ are held constant until the total interior field of Eqs. (37), (50), and (51) takes the form of a standing wave proportional to $\mathbf{M}_{m n}^{(1)}\left(k_{1} \boldsymbol{r}\right)$ and $\mathbf{N}_{m n}^{(1)}\left(k_{1} \boldsymbol{r}\right)$ so as to match the form of the interior wave of Eq. (19) of the full scattering problem of step 1. For the $\mathbf{M}_{m n}^{(1)}\left(k_{1} \boldsymbol{r}\right)$ standing wave this occurs when

$$
\frac{1}{2} I_{m n}^{\mathrm{TE}}=R_{m n}^{11, \mathrm{TE}}+T_{m n}^{21, \mathrm{TE}},
$$

and for the $\mathbf{N}_{m n}^{(1)}\left(k_{1} \boldsymbol{r}\right)$ standing wave it occurs when

$$
\frac{1}{2} I_{m n}^{\mathrm{TM}}=R_{m n}^{11, \mathrm{TM}}+T_{m n}^{21, \mathrm{TM}} .
$$

The same conditions hold for the magnetic field as well. Substituting Eqs. (96) through (99) into Eqs. (102) and (103) and simplifying, one obtains

$$
\begin{aligned}
I_{m_{2} n_{2}}^{\mathrm{TE}}= & \sum_{n=1}^{\infty} \sum_{m=-n}^{+n} \sum_{n_{1}=1}^{\infty} \sum_{m_{1}=-n_{1}}^{+n_{1}} \\
& \times\left[\left(\boldsymbol{I}-\boldsymbol{R}^{11, \mathrm{TE} / \mathrm{TE}}\right)_{m_{2} n_{2}, m n}^{-1} R_{m n, m_{1} n_{1}}^{11, \mathrm{TE} / \mathrm{TM}} I_{m_{1} n_{1}}^{\mathrm{TM}}\right. \\
& +\left(\boldsymbol{I}-\boldsymbol{R}^{11, \mathrm{TE} / \mathrm{TE}}\right)_{m_{2} n_{2}, m n}^{-1} T_{m n, m_{1} n_{1}}^{21, \mathrm{TE}} G_{m_{1} n_{1}}^{\mathrm{TE}} \\
& \left.+\left(\boldsymbol{I}-\boldsymbol{R}^{11, \mathrm{TE} / \mathrm{TE}}\right)_{m_{2} n_{2}, m n}^{-1} T_{m n, m_{1} n_{1}}^{21, \mathrm{TE} / \mathrm{TM}} G_{m_{1} n_{1}}^{\mathrm{TM}}\right],
\end{aligned}
$$


and

$$
\begin{aligned}
I_{m_{2} n_{2}}^{\mathrm{TM}}= & \sum_{n=1}^{\infty} \sum_{m=-n}^{+n} \sum_{n_{1}=1}^{\infty} \sum_{m_{1}=-n_{1}}^{+n_{1}} \\
& \times\left[\left(\boldsymbol{I}-\boldsymbol{R}^{11, \mathrm{TM} / \mathrm{TM}}\right)_{m_{2} n_{2}, m n}^{-1} R_{m n, m_{1} n_{1}}^{11, \mathrm{TM} / \mathrm{TE}} I_{m_{1} n_{1}}^{\mathrm{TE}}\right. \\
& +\left(\boldsymbol{I}-\boldsymbol{R}^{11, \mathrm{TM} / \mathrm{TM}}\right)_{m_{2} n_{2}, m n}^{-1} T_{m n, m_{1} n_{1}}^{21, \mathrm{TM}} G_{m_{1} n_{1}}^{\mathrm{TM}} \\
& \left.+\left(\boldsymbol{I}-\boldsymbol{R}^{11, \mathrm{TM} / \mathrm{TM}}\right)_{m_{2} n_{2}, m n}^{-1} T_{m n, m_{1} n_{1}}^{21, \mathrm{TM} / \mathrm{TE}} G_{m_{1} n_{1}}^{\mathrm{TE}}\right]
\end{aligned}
$$

where $\boldsymbol{I}$ is the identity matrix. The coefficients of $\mathbf{M}_{m n}^{(1)}\left(k_{1} \boldsymbol{r}\right)$ and $\mathbf{N}_{m n}^{(1)}\left(k_{1} \boldsymbol{r}\right)$ obtained in this way are identified as the interior scattering amplitudes $C_{m n}$ and $D_{m n}$ giving

$$
\begin{aligned}
C_{m n}= & I_{m n}^{\mathrm{TM}}=\sum_{n_{1}=1}^{\infty} \sum_{m_{1}=-n_{1}}^{+n_{1}}\left(R_{m n, m_{1} n_{1}}^{11, \mathrm{TM} / \mathrm{TE}} I_{m_{1} n_{1}}^{\mathrm{TE}}+R_{m n, m_{1} n_{1}}^{11, \mathrm{TM} / \mathrm{TM}} I_{m_{1} n_{1}}^{\mathrm{TM}}\right. \\
& \left.+T_{m n, m_{1} n_{1}}^{21, \mathrm{TM} / \mathrm{TE}} G_{m_{1} n_{1}}^{\mathrm{TE}}+T_{m n, m_{1} n_{1}}^{21, \mathrm{TM} / \mathrm{TM}} G_{m_{1} n_{1}}^{\mathrm{TM}}\right), \\
D_{m n}= & I_{m n}^{\mathrm{TE}}=\sum_{n_{1}=1}^{\infty} \sum_{m_{1}=-n_{1}}^{+n_{1}}\left(R_{m n, m_{1} n_{1}}^{11, \mathrm{TE} / \mathrm{TE}} I_{m_{1} n_{1}}^{\mathrm{TE}}+R_{m n, m_{1} n_{1}}^{11, \mathrm{TE} / \mathrm{TM}} I_{m_{1} n_{1}}^{\mathrm{TM}}\right. \\
& \left.+T_{m n, m_{1} n_{1}}^{21, \mathrm{TE} / \mathrm{TE}} G_{m_{1} n_{1}}^{\mathrm{TE}}+T_{m n, m_{1} n_{1}}^{21, \mathrm{TE} / \mathrm{TM}} G_{m_{1} n_{1}}^{\mathrm{TM}}\right) .
\end{aligned}
$$

Equations (104) and (105) form a set of coupled linear equations in the amplitudes $I_{m n}^{\mathrm{TE}}$ and $I_{m n}^{\mathrm{TM}}$. One can solve this set of equations by iteration using the identity

$$
\begin{aligned}
& \left(\boldsymbol{I}-\boldsymbol{R}^{11}\right)_{m_{2} n_{2}, m n}^{-1} \\
& =\delta_{m_{2} m} \delta_{n_{2} n}+R_{m_{2} n_{2}, m n}^{11}+\sum_{n_{3}=1}^{\infty} \sum_{m_{3}=-n_{3}}^{+n_{3}} R_{m_{2} n_{2}, m_{3} n_{3}}^{11} R_{m_{3} n_{3}, m n}^{11} \\
& +\sum_{n_{4}=1}^{\infty} \sum_{m_{4}=-n_{4}}^{+n_{4}} \sum_{n_{3}=1}^{\infty} \sum_{m_{3}=-n_{3}}^{+n_{3}} R_{m_{2} n_{2}, m_{4} n_{4}}^{11} R_{m_{4} n_{4}, m_{3} n_{3}}^{11} R_{m_{3} n_{3}, m n}^{11}+\cdots,
\end{aligned}
$$

assuming that the reflection amplitudes are less than unity. The interior Debye series is obtained by substituting the iteration solution of Eqs. (104) and (105) into Eqs. (106) and (107), with each order of the iteration giving the respective order of the Debye series sum. The results can be decomposed into the polarization-preserving scattering amplitudes $C_{m n, m_{1} n_{1}}^{\mathrm{TM}}$ and $D_{m n, m_{1} n_{1}}^{\mathrm{TE} / \mathrm{TE}}$, and cross-polarized amplitudes $C_{m n, m_{1} n_{1}}^{\mathrm{TM} / \mathrm{TE}}$ and $D_{m n, m_{1} n_{1}}^{\mathrm{TE} / \mathrm{TM}}$ of Eqs. (92) and (93).

Step 5: To evaluate the partial wave scattering $A_{m n}$ and $B_{m n}$ in terms of the partial wave reflection and transmission amplitudes, one now adds the total exterior field of Eqs. (33) and (34) of step 2 to the exterior field of Eq. (54) of step 3 and demands that the result be the combination of a standing wave of the form of Eq. (1) plus an outgoing wave proportional to $\mathbf{M}_{m n}^{(3)}\left(k_{2} \boldsymbol{r}\right)$ and $\mathbf{N}_{m n}^{(3)}\left(k_{2} \boldsymbol{r}\right)$ so as to match the form of the total exterior field of the total problem of Eqs. (1) and (9) of step 1 . The constants of proportionality multiplying $\mathbf{M}_{m n}^{(3)}\left(k_{2} \boldsymbol{r}\right)$ and $\mathbf{N}_{m n}^{(3)}\left(k_{2} \boldsymbol{r}\right)$ are identified as the partial wave scattering amplitudes $B_{m n}$ and $A_{m n}$, respectively. This gives

$$
\begin{gathered}
A_{m n}=-\frac{1}{2} G_{m n}^{\mathrm{TM}}+R_{m n}^{22, \mathrm{TM}}+T_{m n}^{12, \mathrm{TM}}, \\
B_{m n}=-\frac{1}{2} G_{m n}^{\mathrm{TE}}+R_{m n}^{22, \mathrm{TE}}+T_{m n}^{12, \mathrm{TE}} .
\end{gathered}
$$

Substituting Eqs. (94), (95), (100), and (101) into Eqs. (109) and (110) one obtains

$$
\begin{aligned}
A_{m n}= & -\frac{1}{2} G_{m n}^{\mathrm{TM}}+\frac{1}{2} \sum_{n_{1}=1}^{\infty} \sum_{m_{1}=-n_{1}}^{+n_{1}}\left(R_{m n, m_{1} n_{1}}^{22, \mathrm{TM} / \mathrm{TE}} G_{m_{1} n_{1}}^{\mathrm{TE}}\right. \\
& \left.+R_{m n, m_{1} n_{1}}^{22, \mathrm{TM} / \mathrm{TM}} G_{m_{1} n_{1}}^{\mathrm{TM}}+T_{m n, m_{1} n_{1}}^{12, \mathrm{TE}} I_{m_{1} n_{1}}^{\mathrm{TE}}+T_{m n, m_{1} n_{1}}^{12, \mathrm{TM} / \mathrm{TM}} I_{m_{1} n_{1}}^{\mathrm{TM}}\right), \\
B_{m n}= & -\frac{1}{2} G_{m n}^{\mathrm{TE}}+\frac{1}{2} \sum_{n_{1}=1}^{\infty} \sum_{m_{1}=-n_{1}}^{+n_{1}}\left(R_{m n, m_{1} n_{1}}^{22, \mathrm{TE} / \mathrm{TE}} G_{m_{1} n_{1}}^{\mathrm{TE}}\right. \\
& \left.+R_{m n, m_{1} n_{1}}^{22, \mathrm{TE} / \mathrm{TM}} G_{m_{1} n_{1}}^{\mathrm{TM}}+T_{m n, m_{1} n_{1}}^{12, \mathrm{TE} / \mathrm{TE}} I_{m_{1} n_{1}}^{\mathrm{TE}}+T_{m n, m_{1} n_{1}}^{12, \mathrm{TE} / \mathrm{TM}} I_{m_{1} n_{1}}^{\mathrm{TM}}\right) .
\end{aligned}
$$

Now substituting the iteration solution of Eqs. (104) and (105) into Eqs. (111) and (112) one obtains the Debye series of the scattering amplitudes. Again the partial wave amplitudes can be decomposed into the polarization-preserving scattering amplitudes $A_{m n, m_{1} n_{1}}^{\mathrm{TM} / \mathrm{TM}}$ and $B_{m n, m_{1} n_{1}}^{\mathrm{TE} / \mathrm{TE}}$, and cross-polarized amplitudes $A_{m n, m_{1} n_{1}}^{\mathrm{TM} / / \mathrm{TE}}$ and $B_{m n, m_{1} n_{1}}^{\mathrm{TE} / \mathrm{TM}}$ of Eqs. (90) and (91). The formal solution of Eqs. (106), (107), (111), and (112) by inversion and by using recursion relations is given in Appendix B.

As mentioned previously, the iterated form of the interior Debye series is given by substituting Eqs. (104), (105), and (108) into Eqs. (106) and (107). Using the notation of Secs. IV A and IV B, a condensed version of the interior Debye series which emphasizes the individual orders-of-scattering is obtained by collecting together all the terms having the same number of internal reflections, giving

$$
\begin{gathered}
C_{m n}=W_{m n}^{(t), \mathrm{TM}}(0)+\sum_{p=1}^{\infty} W_{m n}^{(r), \mathrm{TM}}(p), \\
D_{m n}=W_{m n}^{(t), \mathrm{TE}}(0)+\sum_{p=1}^{\infty} W_{m n}^{(r), \mathrm{TE}}(p) .
\end{gathered}
$$

Similarly, the iterated form of the exterior Debye series is given by Eqs. (111) and (112). Again collecting together all the terms having the same number of internal reflections and using the notation of Secs. IV A and IV B, we obtain the condensed expressions

$$
\begin{gathered}
A_{m n}=-\frac{1}{2}\left[G_{m n}^{\mathrm{TM}}-W_{m n}^{(r), \mathrm{TM}}(0)-\sum_{p=1}^{\infty} W_{m n}^{(t), \mathrm{TM}}(p)\right], \\
B_{m n}=-\frac{1}{2}\left[G_{m n}^{\mathrm{TE}}-W_{m n}^{(r), \mathrm{TE}}(0)-\sum_{p=1}^{\infty} W_{m n}^{(t), \mathrm{TE}}(p)\right] .
\end{gathered}
$$

These condensed expressions are reminiscent of the structural form of the Debye series for scattering of a plane wave by either a sphere or by a cylinder at normal incidence. But hidden in the $W$ terms via Eqs. (76), (78), (85), (87), and (89) are products of amplitude matrices that are coupled in partial waves, in azimuthal modes, and in polarization state. 


\section{NUMERICAL VERIFICATION}

To numerically verify the correctness of the EBCM Debye series developed in Sec. III for electromagnetic scattering by a nonspherical particle, we computed the $p=0,1,2$ scattering contributions for a plane wave with wavelength $\lambda=0.5145 \mu \mathrm{m}$ traveling in the $Z$ direction and vertically polarized in the $Y$ direction incident on an oblate spheroidal water droplet with an equivalent-volume sphere radius of $10.0 \mu \mathrm{m}$, horizontal-to-vertical radius ratio $b / a=1.25,1.31$, and refractive index $m=1.334+1.2 \times 10^{-9} i$. The laboratory coordinate system $O X Y Z$ for an oblate spheroid illuminated by the plane wave is illustrated in Fig. 2. These parameters were chosen because the $p=2$ Debye series component of this system has already been extensively studied using VSM Debye series in oblate spheroidal coordinates [46]. Thus a comparison of the results of the procedure described in Sec. III with published results may be readily made. The results of our numerical computations are shown in Figs. 3 through 6. In the polar plots of Figs. 3 through 6, the scattering angle $\Theta$ (varying in the radial direction) is measured with respect to the beam propagation direction while the azimuthal scattering angle $\Phi$ (varying in the angular direction in the figures) is measured with respect to the equatorial plane of the oblate droplet. These angles are obtained from a conversion of the spherical coordinate angles defined in Sec. II.

The $p=0$ scattering contribution of Fig. 3(a) is dominated by diffraction. For the radius ratio $b / a=1.25$, the horizontal radius of the spheroidal droplet is $b=10.77 \mu \mathrm{m}$. The diffraction structure should be well-approximated by the Fraunhofer diffraction pattern of Refs. $[47,48]$ which in the $\Phi=0^{\circ}$ horizontal direction should have its first two minima at $\Theta=1.70^{\circ}$ and $3.06^{\circ}$. In the $\Phi=90^{\circ}$ vertical direction, the Fraunhofer minima are predicted to be at $\Theta=2.09^{\circ}$ and $3.82^{\circ}$. These predictions agree well with Fig. 3.

The $p=1$ plot for transmission in Fig. 4(a) exhibits the monotonic falloff of the scattered intensity as a function of $\Theta$ for all $\Phi$ due to the progressive decrease in the Fresnel coefficients for transmission into and out of the particle, the Fock transition in the vicinity of the grazing incident ray having the critical internal angle for total reflection, and the exponential decrease in the intensity thereafter due to electromagnetic surface waves created at the total internal reflection locations on the particle surface. The horizontal radius of the particle is again $b=10.77 \mu \mathrm{m}$ giving a size parameter of $k_{2} b=131.55$. For scattering in the horizontal

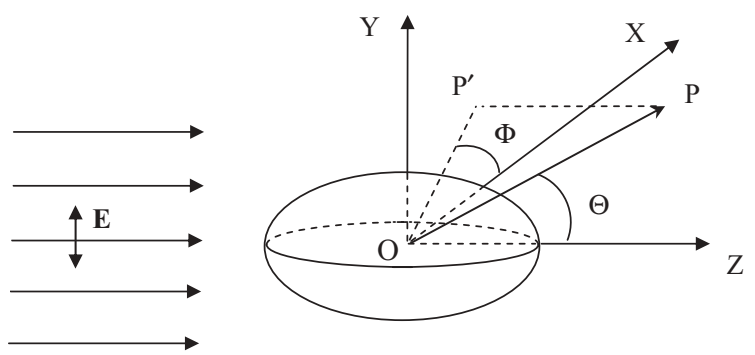

FIG. 2. Geometry of the laboratory coordinates for an oblate spheroid of semimajor axis $b$ and semiminor axis $a$ is illuminated by a side-on incident plane wave with electric field component polarized in the $Y$ direction.

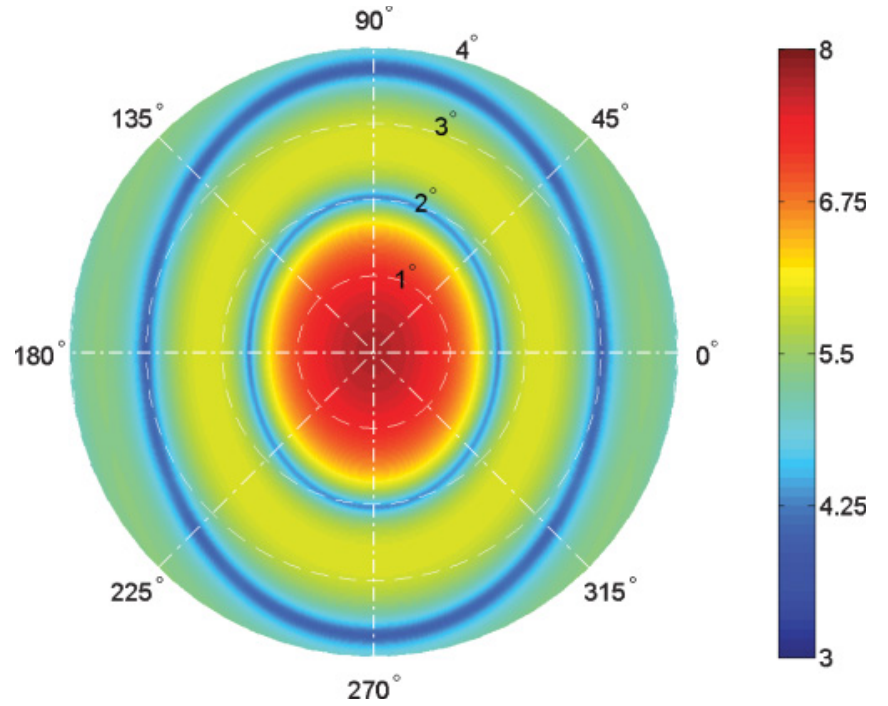

(a)

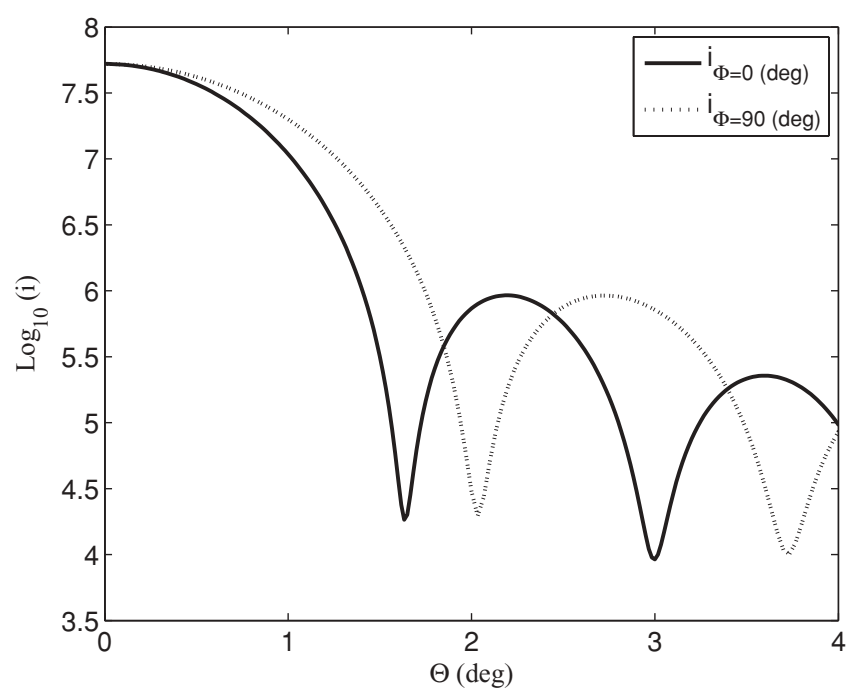

(b)

FIG. 3. (Color online) (a) Polar plot of near forward scattered intensities of order $p=0$ as a function of the scattering angle $\Theta$ and the azimuthal angle $\Phi$ for a vertically polarized incident plane wave with wavelength $\lambda=0.5145 \mu \mathrm{m}$ scattered by an oblate spheroid of refractive index $m=1.334+1.2 \times 10^{-9} i$, equivalent-volume sphere radius $10.0 \mu \mathrm{m}$, and horizontal-to-vertical radius ratio $b / a=1.25$. The intensities are plotted in a common logarithm scale. (b) Twodimensional plot of the intensities in the equatorial plane $\left(\Phi=0^{\circ}\right)$ and vertical plane $\left(\Phi=90^{\circ}\right)$ versus the scattering angle.

plane with $\Phi=0^{\circ}$, the scattering angle of the grazing incident ray is $\Theta=82.88^{\circ}$. The $\Phi=0^{\circ}$ intensity of Fig. 4(b) closely matches the intensity for $k_{2} b=100$ of Fig. 2(b) of Ref. [49] for scattering by a sphere. The small difference between the spheroid and sphere cases is likely due to the fact that for scattering by a sphere each ray path is confined to a single plane. The intensity of Fig. 2(b)of Ref. [49] is then due only to rays incident in the horizontal plane. But the plane of incidence of a given ray for scattering by a spheroid changes at each interaction of the ray with the spheroid surface. This produces small contributions in the horizontal scattering plane from 


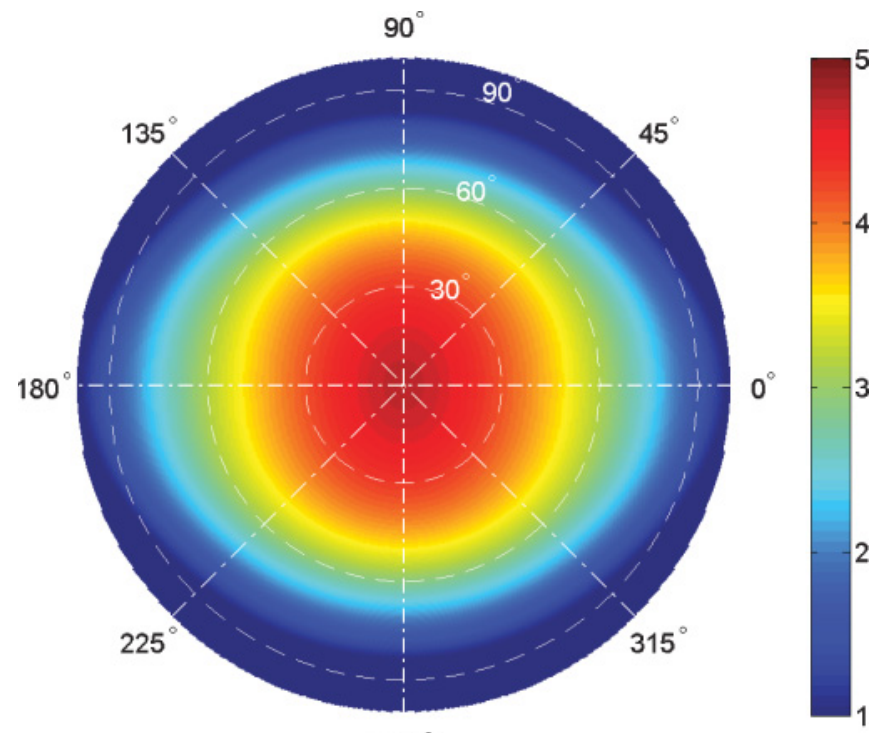

$270^{\circ}$

(a)

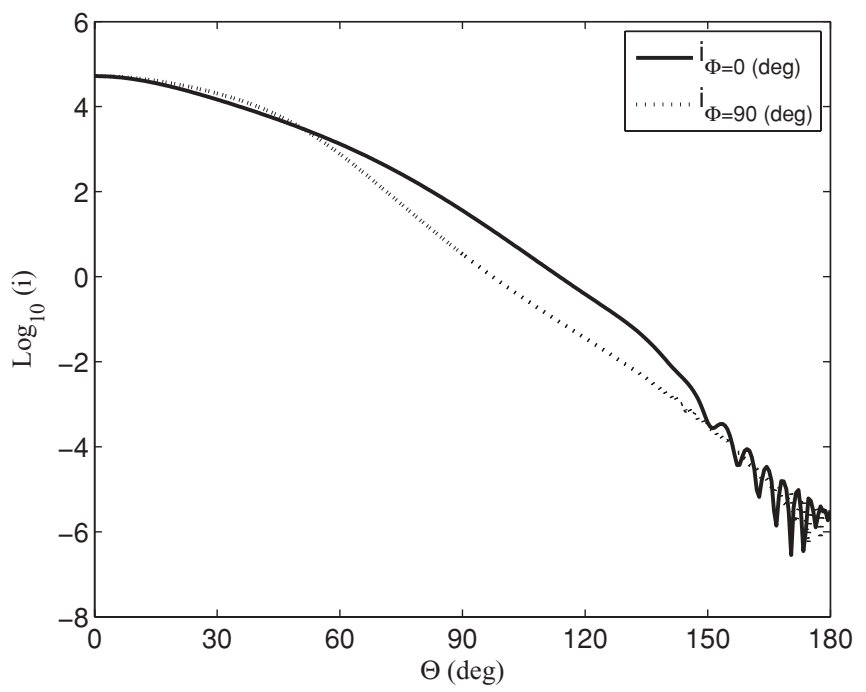

(b)

FIG. 4. (Color online) Same as Fig. 3 but for $p=1$-order scattered intensities at the angles (a) $0^{\circ} \leqslant \Theta \leqslant 100^{\circ}$ and (b) $0^{\circ} \leqslant$ $\Theta \leqslant 180^{\circ}$.

skew rays that were incident on the spheroid in other planes. For scattering by a spheroid in the $\Phi=90^{\circ}$ vertical plane, two effects occur that tend to compensate for each other. First, when the grazing incident ray inside the particle approaches the $p=1$ interaction with the particle surface, the internal transmission angle is smaller than the critical angle. Thus an increasing portion of the wave is transmitted out of the particle leading to a slower attenuation of the transmitted intensity as a function of $\Theta$ than for a sphere. Second, at the $p=0$ interaction of the grazing ray with the spheroid surface, the radius of curvature of the surface is now $b(b / a)$ rather than $b$, as was the case for a sphere. As a result of this increased radius of curvature, the incoming grazing ray sees an effectively larger size particle, the surface wave created at the $p=0$ interface dies off faster, and the resulting scattered intensity as a function of $\Theta$ is attenuated at a faster rate than for a sphere. In Fig. 4(b),

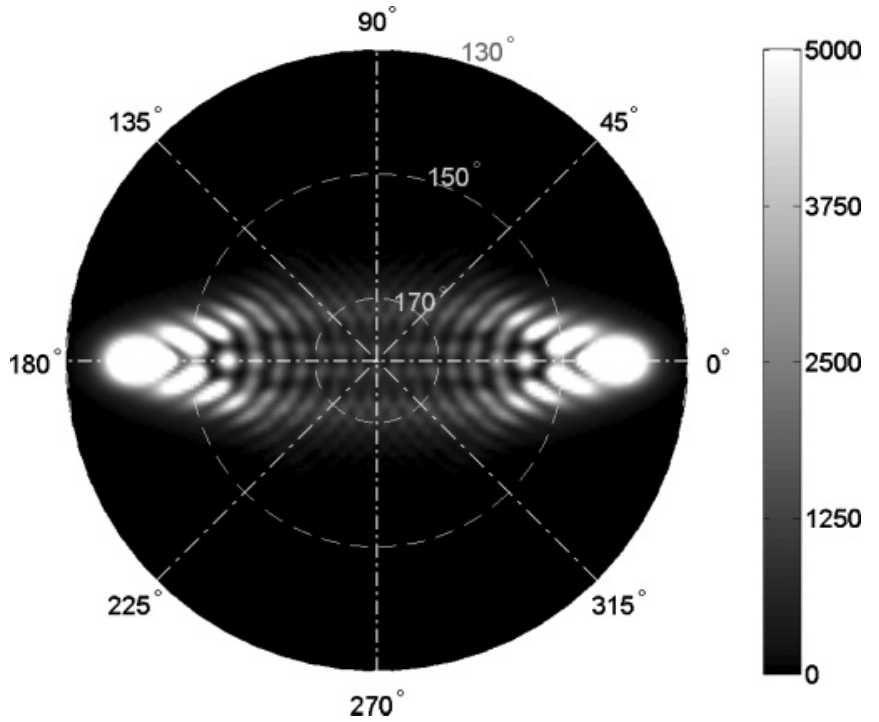

FIG. 5. Same as Fig. 3(a) but for $p=2$-order scattered intensities at the angles $130^{\circ} \leqslant \Theta \leqslant 180^{\circ}$. The intensities are plotted on a linear scale.

it is seen that the increasing angular attenuation effect is somewhat larger than the decreasing angular attenuation effect.

The $p=2$ plots of Figs. 5 and 6 for $b / a=1.25$ with $b=10.77 \mu \mathrm{m}$, and $b / a=1.31$ with $b=10.94 \mu \mathrm{m}$ closely match Figs. 5(d) and 5(e) of Ref. [46], which were computed for $b=6.00 \mu \mathrm{m}$ (or $a=6.00 \mu \mathrm{m}$ in Ref. [46]) using the VSM Debye series in spheroidal coordinates. More detail of the interference structure is visible in Figs. 5 and 6 here than was the case in Figs. 4(d) and Fig. 4(e) of Ref. [46] since the spheroid is somewhat larger than it was before, the angular size of the diffraction structure is correspondingly smaller, so corresponding more of it can fit into a given angular interval. This agreement for $p=0,1,2$, along with the fact that the EBCM formalism is capable of being used for larger particle sizes than the spheroidal coordinate VSM formulism can, underscores the predictive power of the EBCM Debye series.

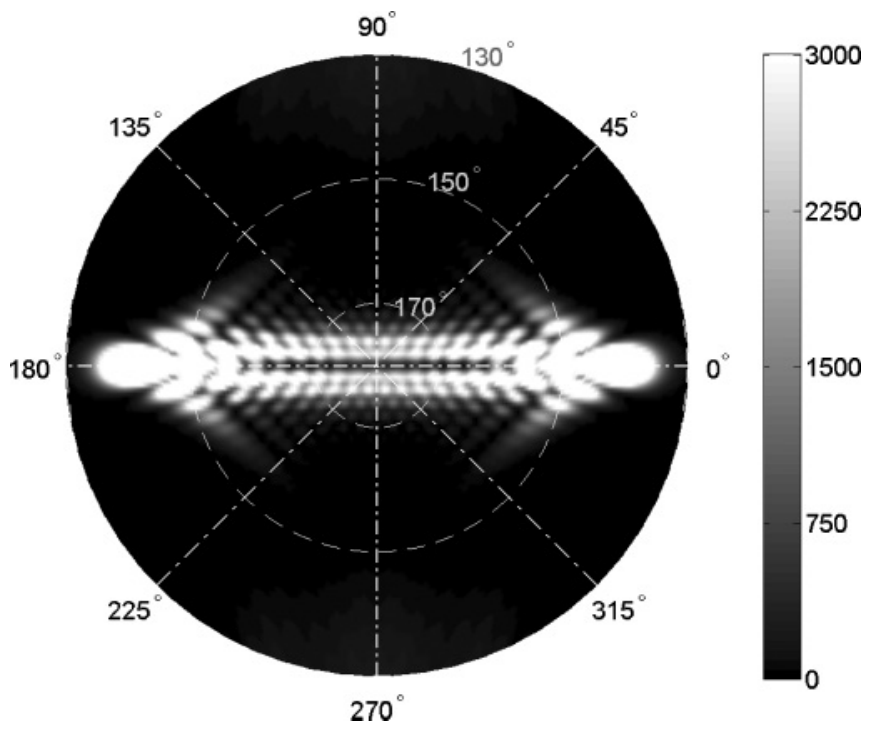

FIG. 6. Same as Fig. 5 but for $b / a=1.31$. 


\section{CONCLUSION}

The original version of the Debye series, first derived by Peter Debye in [1908], decomposed the partial wave amplitudes for scattering of a plane wave by a homogeneous dielectric circular cylinder at normal incidence into the effects produced by diffraction, external reflection, and transmission following $(p-1)$ internal reflections. This is the wave theory analog of scattering by geometrical light rays, and in the short wavelength limit the Debye series results have been shown to approach the corresponding ray theory results. Since the time of Debye, his series has been generalized to scattering of a plane wave or an arbitrary incident beam by a plethora of high-symmetry particles such as a sphere, a coated sphere, a multilayer sphere, a circular cylinder at diagonal incidence, and a spheroid. In each case the total scattering can again be decomposed into the contributions produced by diffraction, external reflection, and transmission following all numbers of internal reflections. This pattern leads one to believe that this decomposition is universal, and that as a result, when the scattering and interior amplitudes are partitioned into this particular form, there are no additional physical process waiting to be discovered in scattering by a homogeneous particle having a more general shape. This is not to mean that transmissions or reflections of light from such particles will not produce novel and unexpected cooperative effects. It only means that such cooperative effects should be capable of being understood in terms of the transmission and reflection amplitudes described in this article. The verification of the Debye series described in detail here shows that this indeed will be the case as long as the particle in question is homogeneous with a well-defined outer surface. Our verification uses an extension of the EBCM formalism which treats light scattering in spherical coordinates independent of the details of the shape of the particle's surface. Thus no special coordinate system in which the vector wave equation is separable is required to match the boundary conditions of the electric and magnetic fields at the particle surface. The pertinent Debye series EBCM equations are enumerated in the last paragraph of Sec. I. In future studies we will continue the program of numerically examining and physically interpreting the Debye decomposition of light scattering by various nonspherical particles in a number of practical situations. Lastly, it should be noted that the generalization of Debye series for a multilayer particle or a particle with continuously varying refractive index, and fully understanding all the ramifications of the Debye series expansion for an inhomogeneous particle, need further efforts.

\section{ACKNOWLEDGMENTS}

This research is supported by the German Research Foundation (Grant No. TR194/49-1) and the Alexander von Humboldt Foundation (Grant No. CHN/1127072). F. Xu thanks Prof. Cameron Tropea at the Institute of Fluid Mechanics and Aerodynamics of Technical University of Darmstadt for the great encouragement he has shown in our pursuit of this work, and for many fruitful conversations concerning it.

\section{APPENDIX A: DEFINITION OF VSWF'S IN GLMT AND EBCM}

In GLMT the incident field is determined from the Bromwich scalar potentials using derivative rules [9]. Assuming $E_{0}$ to be unity, the electric field can be expressed by

$$
\begin{aligned}
E_{r}^{i}= & \frac{1}{k r^{2}} \sum_{n=1}^{+\infty} \sum_{m=-n}^{+n} c_{n}^{\mathrm{pw}} g_{m n}^{\mathrm{TM}} n(n+1) \\
& \times \psi_{n}(k r) P_{|m| n}(\cos \theta) \exp (i m \phi), \\
E_{\theta}^{i}= & \frac{1}{r} \sum_{n=1}^{+\infty} \sum_{m=-n}^{+n} c_{n}^{\mathrm{pw}}\left[g_{m n}^{\mathrm{TE}} m \psi_{n}(k r) \frac{P_{|m| n}(\cos \theta)}{\sin \theta}\right. \\
& \left.+g_{m n}^{\mathrm{TM}} \psi_{n}^{\prime}(k r) \frac{d P_{|m| n}(\cos \theta)}{d \theta}\right] \exp (i m \phi), \\
E_{\phi}^{i}= & \frac{i}{r} \sum_{n=1}^{+\infty} \sum_{m=-n}^{+n} c_{n}^{\mathrm{pw}}\left[g_{m n}^{\mathrm{TE}} \psi_{n}(k r) \frac{d P_{|m| n}(\cos \theta)}{d \theta}\right. \\
& \left.+g_{m n}^{\mathrm{TM}} m \psi_{n}^{\prime}(k r) \frac{P_{|m| n}(\cos \theta)}{\sin \theta}\right] \exp (i m \phi),
\end{aligned}
$$

where $c_{n}^{\mathrm{pw}}$ is defined by Eq. (8), the Riccatti-Bessel function $\psi_{n}(x)$ is related to the spherical Bessel function $j_{n}(x)$ by $\psi_{n}(x)=x j_{n}(x)$, and $P_{m n}$ is the associated Legendre function using Hobson's definition

$$
P_{m n}(x)=(-1)^{m}\left(1-x^{2}\right)^{\frac{m}{2}} \frac{d^{m}}{d x^{m}} P_{n}(x) .
$$

However, Eqs. (A1) through (A3) use the time dependence $e^{i \omega t}$. When the time dependence $e^{-i \omega t}$ is assumed instead, we have

$$
\begin{aligned}
E_{r}^{i}= & \frac{1}{k r^{2}} \sum_{n=1}^{+\infty} \sum_{m=-n}^{+n} \bar{c}_{n}^{\mathrm{pw}} \bar{g}_{m n}^{\mathrm{TM}} n(n+1) \\
& \times \psi_{n}(k r) P_{|m| n}(\cos \theta) \exp (-i m \phi), \\
E_{\theta}^{i}= & \frac{1}{r} \sum_{n=1}^{+\infty} \sum_{m=-n}^{+n} \bar{c}_{n}^{\mathrm{pw}}\left[\bar{g}_{m n}^{\mathrm{TE}} m \psi_{n}(k r) \frac{P_{|m| n}(\cos \theta)}{\sin \theta}\right. \\
& \left.+\bar{g}_{m n}^{\mathrm{TM}} \psi_{n}^{\prime}(k r) \frac{d P_{|m| n}(\cos \theta)}{d \theta}\right] \exp (-i m \phi), \\
E_{\phi}^{i}= & -\frac{i}{r} \sum_{n=1}^{+\infty} \sum_{m=-n}^{+n} \bar{c}_{n}^{\mathrm{pw}}\left[\bar{g}_{m n}^{\mathrm{TE}} \psi_{n}(k r) \frac{d P_{|m| n}(\cos \theta)}{d \theta}\right. \\
& \left.+\bar{g}_{m n}^{\mathrm{TM}} m \psi_{n}^{\prime}(k r) \frac{P_{|m| n}(\cos \theta)}{\sin \theta}\right] \exp (-i m \phi),
\end{aligned}
$$

where the bar over $g$ and $c$ denotes complex conjugation. Replacing $m$ by $-m$ and defining the VSWF's $\mathbf{M}_{m n}^{(1), G}$ and $\mathbf{N}_{m n}^{(1), G}$ as follows for GLMT

$$
\begin{aligned}
\mathbf{M}_{m n}^{(1), G}(k r, \theta, \varphi)= & {\left[i m j_{n}(k r) \frac{P_{|m| n}(\cos \theta)}{\sin \theta} \mathbf{i}_{\theta}\right.} \\
& \left.-j_{n}(k r) \frac{d P_{|m| n}(\cos \theta)}{d \theta} \mathbf{i}_{\varphi}\right] \exp (i m \phi),
\end{aligned}
$$




$$
\begin{aligned}
\mathbf{N}_{m n}^{(1), G}(k r, \theta, \varphi)= & \frac{1}{k r}\left[n(n+1) j_{n}(k r) P_{|m| n}(\cos \theta) \mathbf{i}_{r}\right. \\
& +\frac{d\left(k r j_{n}(k r)\right)}{d(k r)} \frac{d P_{|m| n}(\cos \theta)}{d \theta} \mathbf{i}_{\theta} \\
& \left.+i m \frac{d\left(k r j_{n}(k r)\right)}{d(k r)} \frac{P_{|m| n}(\cos \theta)}{\sin \theta} \mathbf{i}_{\varphi}\right] \exp (i m \phi),
\end{aligned}
$$

the incident electric field can be rewritten in terms of VSWF's

$$
\mathbf{E}^{(i)}=\sum_{n=1}^{+\infty} \sum_{m=-n}^{+n} k \bar{c}_{n}^{\mathrm{pw}}\left[\left(i \bar{g}_{-m n}^{\mathrm{TE}}\right) \mathbf{M}_{m n}^{(1), G}+\bar{g}_{-m n}^{\mathrm{TM}} \mathbf{N}_{m n}^{(1), G}\right] .
$$

Substituting Eq. (8) into Eq. (A10) we have

$$
\begin{aligned}
\mathbf{E}^{(i)}= & \sum_{n=1}^{+\infty} \sum_{m=-n}^{+n}\left\{\left[k \frac{(2 n+1) i^{n+1}}{n(n+1)} i \bar{g}_{-m n}^{\mathrm{TE}}\right] \mathbf{M}_{m n}^{(1), G}\right. \\
& \left.+\left[k \frac{(2 n+1) i^{n+1}}{n(n+1)} \bar{g}_{-m n}^{\mathrm{TM}}\right] \mathbf{N}_{m n}^{(1), G}\right\} .
\end{aligned}
$$

In the $T$-matrix formulation of Ref. [31], however, $\mathbf{M}_{m n}^{(1), T}$ and $\mathbf{N}_{m n}^{(1), T}$ are defined by

$$
\begin{aligned}
\mathbf{M}_{m n}^{(1), T}(k r, \theta, \varphi)= & \gamma_{m n}\left[i m j_{n}(k r) \frac{P_{m n}(\cos \theta)}{\sin \theta} \mathbf{i}_{\theta}\right. \\
& \left.-j_{n}(k r) \frac{d P_{m n}(\cos \theta)}{d \theta} \mathbf{i}_{\varphi}\right] \exp (i m \phi), \\
\mathbf{N}_{m n}^{(1), T}(k r, \theta, \varphi)= & \frac{\gamma_{m n}}{k r}\left[n(n+1) j_{n}(k r) P_{m n}(\cos \theta) \mathbf{i}_{r}\right. \\
& +\frac{d\left(k r j_{n}(k r)\right)}{d(k r)} \frac{d P_{m n}(\cos \theta)}{d \theta} \mathbf{i}_{\theta} \\
& \left.+i m \frac{d\left(k r j_{n}(k r)\right)}{d(k r)} \frac{P_{m n}(\cos \theta)}{\sin \theta} \mathbf{i}_{\varphi}\right] \exp (i m \phi),
\end{aligned}
$$

where

$$
\gamma_{m n}=\left[\frac{(2 n+1)(n-m) !}{4 \pi n(n+1)(n+m) !}\right]^{1 / 2} .
$$

Comparison of the two definitions of VSWF's shows the following relation

$$
\begin{array}{r}
\mathbf{M}_{m n}^{(1), T}=F_{m n} \mathbf{M}_{m n}^{(1), G}, \\
\mathbf{N}_{m n}^{(1), T}=F_{m n} \mathbf{N}_{m n}^{(1), G},
\end{array}
$$

where the connection factor $F_{m n}$ is expressed by Eq. (5). Using the VSWF's defined in Ref. [31] and substituting Eqs. (A15) and (A16) into Eq. (A11), Eq. (1) can be obtained. Note that the superscript " $T$ " is dropped in the main body of the article.

\section{APPENDIX B: FORMAL AND RECURSION SOLUTIONS OF THE DEBYE SERIES}

A formal summation of the interior Debye series can be obtained by the inversion of Eqs. (106) and (107). These equations can be written as a set of coupled matrix equations as

$$
\begin{aligned}
\boldsymbol{C}= & \boldsymbol{R}^{11, \mathrm{TM} / \mathrm{TE}} \boldsymbol{D}+\boldsymbol{R}^{11, \mathrm{TM} / \mathrm{TM}} \boldsymbol{C}+\boldsymbol{T}^{21, \mathrm{TM} / \mathrm{TE}} \boldsymbol{G}^{\mathrm{TE}} \\
& +\boldsymbol{T}^{21, \mathrm{TM} / \mathrm{TM}} \boldsymbol{G}^{\mathrm{TM}} \\
\boldsymbol{D}= & \boldsymbol{R}^{11, \mathrm{TE} / \mathrm{TE}} \boldsymbol{D}+\boldsymbol{R}^{11, \mathrm{TE} / \mathrm{TM}} \boldsymbol{C}+\boldsymbol{T}^{21, \mathrm{TE} / \mathrm{TE}} \boldsymbol{G}^{\mathrm{TE}} \\
& +\boldsymbol{T}^{21, \mathrm{TE} / \mathrm{TM}} \boldsymbol{G}^{\mathrm{TM}} .
\end{aligned}
$$

This makes implicit the coupling of partial waves and azimuthal modes in the off-diagonal elements of the matrices and focuses on the coupling of the polarization states. We define the matrices $\boldsymbol{S}, \boldsymbol{U}$, and $\boldsymbol{V}$ as

$$
\begin{aligned}
& \boldsymbol{S}^{\mathrm{TE} / \mathrm{TE}}=\left(\boldsymbol{I}-\boldsymbol{R}^{11, \mathrm{TE} / \mathrm{TE}}\right)^{-1}, \\
& \boldsymbol{S}^{\mathrm{TM} / \mathrm{TM}}=\left(\boldsymbol{I}-\boldsymbol{R}^{11, \mathrm{TM} / \mathrm{TM}}\right)^{-1}, \\
& \boldsymbol{U}^{\mathrm{TE} / \mathrm{TE}}=\left(\boldsymbol{I}-\boldsymbol{S}^{\mathrm{TE} / \mathrm{TE}} \boldsymbol{R}^{11, \mathrm{TE} / \mathrm{TM}} \boldsymbol{S}^{\mathrm{TM} / \mathrm{TM}} \boldsymbol{R}^{11, \mathrm{TM} / \mathrm{TE}}\right)^{-1}, \\
& \boldsymbol{U}^{\mathrm{TM} / \mathrm{TM}}=\left(\boldsymbol{I}-\boldsymbol{S}^{\mathrm{TM} / \mathrm{TM}} \boldsymbol{R}^{11, \mathrm{TM} / \mathrm{TE}} \boldsymbol{S}^{\mathrm{TE} / \mathrm{TE}} \boldsymbol{R}^{11, \mathrm{TE} / \mathrm{TM}}\right)^{-1}, \\
& \boldsymbol{V}^{\mathrm{TE} / \mathrm{TE}}=\boldsymbol{R}^{11, \mathrm{TE} / \mathrm{TM}} \boldsymbol{S}^{\mathrm{TM} / \mathrm{TM}} \boldsymbol{T}^{21, \mathrm{TM} / \mathrm{TE}}+\boldsymbol{T}^{21, \mathrm{TE} / \mathrm{TE}}, \\
& \boldsymbol{V}^{\mathrm{TE} / \mathrm{TM}}=\boldsymbol{R}^{11, \mathrm{TE} / \mathrm{TM}} \boldsymbol{S}^{\mathrm{TM} / \mathrm{TM}} \boldsymbol{T}^{21, \mathrm{TM} / \mathrm{TM}}+\boldsymbol{T}^{21, \mathrm{TE} / \mathrm{TM}}, \\
& \boldsymbol{V}^{\mathrm{TM} / \mathrm{TE}}=\boldsymbol{R}^{11, \mathrm{TM} / \mathrm{TE}} \boldsymbol{S}^{\mathrm{TE} / \mathrm{TE}} \boldsymbol{T}^{21, \mathrm{TE} / \mathrm{TE}}+\boldsymbol{T}^{21, \mathrm{TM} / \mathrm{TE}}, \\
& \boldsymbol{V}^{\mathrm{TM} / \mathrm{TM}}=\boldsymbol{R}^{11, \mathrm{TM} / \mathrm{TE}} \boldsymbol{S}^{\mathrm{TE} / \mathrm{TE}} \boldsymbol{T}^{21, \mathrm{TE} / \mathrm{TM}}+\boldsymbol{T}^{21, \mathrm{TM} / \mathrm{TM}} .
\end{aligned}
$$

Then substituting Eq. (B2) into Eq. (B1) and decomposing the result into the form of Eqs. (95) and (96), we obtain

$$
\begin{gathered}
\boldsymbol{C}^{\mathrm{TM} / \mathrm{TE}}=\boldsymbol{U}^{\mathrm{TM} / \mathrm{TM}} \boldsymbol{S}^{\mathrm{TM} / \mathrm{TM}} \boldsymbol{V}^{\mathrm{TM} / \mathrm{TE}}, \\
\boldsymbol{C}^{\mathrm{TM} / \mathrm{TM}}=\boldsymbol{U}^{\mathrm{TM} / \mathrm{TM}} \boldsymbol{S}^{\mathrm{TM} / \mathrm{TM}} \boldsymbol{V}^{\mathrm{TM} / \mathrm{TM}}, \\
\boldsymbol{D}^{\mathrm{TE} / \mathrm{TE}}=\boldsymbol{U}^{\mathrm{TE} / \mathrm{TE}} \boldsymbol{S}^{\mathrm{TE} / \mathrm{TE}} \boldsymbol{V}^{\mathrm{TE} / \mathrm{TE}}, \\
\boldsymbol{D}^{\mathrm{TE} / \mathrm{TM}}=\boldsymbol{U}^{\mathrm{TE} / \mathrm{TE}} \boldsymbol{S}^{\mathrm{TE} / \mathrm{TE}} \boldsymbol{V}^{\mathrm{TE} / \mathrm{TM}},
\end{gathered}
$$

for the interior Debye series.

A formal summation of the Debye series of the scattering amplitudes can then be obtained from the interior amplitude solution. We write Eqs. (111) and (112) as matrix equations, substitute Eqs. (106) and (107), and decompose the result in the form of Eqs. (90) and (91). We obtain

$$
\begin{aligned}
\boldsymbol{A}^{\mathrm{TM} / \mathrm{TE}}= & \frac{1}{2}\left(\boldsymbol{R}^{22, \mathrm{TM} / \mathrm{TE}}+\boldsymbol{T}^{12, \mathrm{TM} / \mathrm{TE}} \boldsymbol{D}^{\mathrm{TE} / \mathrm{TE}}\right. \\
& \left.+\boldsymbol{T}^{12, \mathrm{TM} / \mathrm{TM}} \boldsymbol{C}^{\mathrm{TM} / \mathrm{TE}}\right) \\
\boldsymbol{A}^{\mathrm{TM} / \mathrm{TM}}= & -\frac{1}{2}\left(\boldsymbol{I}-\boldsymbol{R}^{22, \mathrm{TM} / \mathrm{TM}}-\boldsymbol{T}^{12, \mathrm{TM} / \mathrm{TE}} \boldsymbol{D}^{\mathrm{TE} / \mathrm{TM}}\right. \\
& \left.-\boldsymbol{T}^{12, \mathrm{TM} / \mathrm{TM}} \boldsymbol{C}^{\mathrm{TM} / \mathrm{TM}}\right) \\
\boldsymbol{B}^{\mathrm{TE} / \mathrm{TE}}= & -\frac{1}{2}\left(\boldsymbol{I}-\boldsymbol{R}^{22, \mathrm{TE} / \mathrm{TE}}-\boldsymbol{T}^{12, \mathrm{TE} / \mathrm{TE}} \boldsymbol{D}^{\mathrm{TE} / \mathrm{TE}}\right. \\
& \left.-\boldsymbol{T}^{12, \mathrm{TE} / \mathrm{TM}} \boldsymbol{C}^{\mathrm{TM} / \mathrm{TE}}\right) \\
\boldsymbol{B}^{\mathrm{TE} / \mathrm{TM}}= & \frac{1}{2}\left(\boldsymbol{R}^{22, \mathrm{TE} / \mathrm{TM}}+\boldsymbol{T}^{12, \mathrm{TE} / \mathrm{TE}} \boldsymbol{D}^{\mathrm{TE} / \mathrm{TM}}\right. \\
& \left.+\boldsymbol{T}^{12, \mathrm{TE} / \mathrm{TM}} \boldsymbol{C}^{\mathrm{TM} / \mathrm{TM}}\right) .
\end{aligned}
$$

Diffraction (i.e., the identity matrix term) occurs only in the polarization-preserving channels, external reflection occurs in 
all polarization channels, and the last two terms of Eqs. (B15) through (B18) describe light already in the particle that must be transmitted back out. Substitution of Eqs. (B11) through (B14) to Eqs. (B15) through (B18) gives the formal summation of the Debye series of the scattering amplitudes.

These results can be recast in the order-of-scattering formalism to provide a recursive generation of the Debye series. Let

$$
\begin{aligned}
& \boldsymbol{C}^{i / j}=\sum_{p=0}^{\infty} \boldsymbol{C}_{p}^{i / j}, \\
& \boldsymbol{D}^{i / j}=\sum_{p=0}^{\infty} \boldsymbol{D}_{p}^{i / j},
\end{aligned}
$$

for the interior amplitudes where $i$ and $j$ are each either TE or TM, and

$$
\begin{aligned}
\boldsymbol{A}^{i / j} & =\sum_{p=0}^{\infty} \boldsymbol{A}_{p}^{i / j}, \\
\boldsymbol{B}^{i / j} & =\sum_{p=0}^{\infty} \boldsymbol{B}_{p}^{i / j},
\end{aligned}
$$

for the scattering amplitudes where the integer $p$ parameterizes the number of internal reflections that have occurred to that point in the series. For the interior field, from Eqs. (104) through (107), one has the starting conditions

$$
\boldsymbol{C}_{0}^{\mathrm{TM} / j}=\boldsymbol{T}^{21, \mathrm{TM} / j},
$$

$$
\boldsymbol{D}_{0}^{\mathrm{TE} / j}=\boldsymbol{T}^{21, \mathrm{TE} / j},
$$

where $j$ is either TE or TM, and the coupled recursion relations

$$
\left[\begin{array}{l}
\boldsymbol{D}_{p}^{\mathrm{TE} / j} \\
\boldsymbol{C}_{p}^{\mathrm{TM} / j}
\end{array}\right]=\left[\begin{array}{ll}
\boldsymbol{R}^{11, \mathrm{TE} / \mathrm{TE}} & \boldsymbol{R}^{11, \mathrm{TE} / \mathrm{TM}} \\
\boldsymbol{R}^{11, \mathrm{TM} / \mathrm{TE}} & \boldsymbol{R}^{11, \mathrm{TM} / \mathrm{TM}}
\end{array}\right]\left[\begin{array}{c}
\boldsymbol{D}_{p-1}^{\mathrm{TE} / j} \\
\boldsymbol{C}_{p-1}^{\mathrm{TM} / j}
\end{array}\right],
$$

for $p \geqslant 1$, which are equivalent to Eqs. (113) and (114). The $p$-order interior amplitudes thus contain $p$ internal reflections. Similarly, from Eqs. (104), (105), (108), (111), and (112) the $p$-order scattering amplitudes are given by the starting conditions

$$
\begin{gathered}
\boldsymbol{A}_{0}^{\mathrm{TM} / \mathrm{TE}}=\frac{1}{2} \boldsymbol{R}^{22, \mathrm{TM} / \mathrm{TE}}, \\
\boldsymbol{A}_{0}^{\mathrm{TM} / \mathrm{TM}}=-\frac{1}{2}\left(\boldsymbol{I}-\boldsymbol{R}^{22, \mathrm{TM} / \mathrm{TM}}\right), \\
\boldsymbol{B}_{0}^{\mathrm{TE} / \mathrm{TE}}=-\frac{1}{2}\left(\boldsymbol{I}-\boldsymbol{R}^{22, \mathrm{TE} / \mathrm{TE}}\right), \\
\boldsymbol{B}_{0}^{\mathrm{TE} / \mathrm{TM}}=\frac{1}{2} \boldsymbol{R}^{22, \mathrm{TE} / \mathrm{TM}},
\end{gathered}
$$

and the recursion relations using the $(p-1)$-order interior amplitudes as input are

$$
\left[\begin{array}{c}
\boldsymbol{B}_{p}^{\mathrm{TE} / j} \\
\boldsymbol{A}_{p}^{\mathrm{TM} / j}
\end{array}\right]=\left[\begin{array}{cc}
\boldsymbol{T}^{12, \mathrm{TE} / \mathrm{TE}} & \boldsymbol{T}^{12, \mathrm{TE} / \mathrm{TM}} \\
\boldsymbol{T}^{12, \mathrm{TM} / \mathrm{TE}} & \boldsymbol{T}^{12, \mathrm{TM} / \mathrm{TM}}
\end{array}\right]\left[\begin{array}{c}
\boldsymbol{D}_{p-1}^{\mathrm{TE} / j} \\
\boldsymbol{C}_{p-1}^{\mathrm{TM} / j}
\end{array}\right],
$$

for $p \geqslant 1$, which are equivalent to Eqs. (115) and (116). The $p$-order scattering amplitudes now contain $(p-1)$ internal reflections, and both polarization-preserving and cross-polarized effects occur at every step along the way.
[1] G. Mie, Ann. Phys. (Leipzig) 25, 377 (1908).

[2] N. Morita, T. Tanaka, T. Yamasaki, and Y. Nakanishi, IEEE Trans. Antennas Propag. 16, 724 (1968).

[3] W. C. Tsai and R. J. Pogorzelski, J. Opt. Soc. Am. 65, 1457 (1975).

[4] W. G. Tam and R. Corriveau, J. Opt. Soc. Am. 68, 763 (1978).

[5] G. Gouesbet, J. Quant. Spectrosc. Radiat. Transfer 110, 1223 (2009).

[6] J. A. Lock and G. Gouesbet, J. Quant. Spectrosc. Radiat. Transfer 110, 800 (2009).

[7] T. Wriedt, Part. Part. Syst. Charact. 15, 67 (1998).

[8] P. C. Waterman, Phys. Rev. D 3, 825 (1971).

[9] G. Gouesbet, Opt. Commun. 283, 517 (2010).

[10] M. I. Mishchenko, L. D. Travis, and A. A. Lacis, Scattering, Absorption, and Emission of Light by Small Particles (Cambridge University Press, Cambridge, England, 2002), Chap. 5.

[11] P. W. Barber and S. C. Hill, Light Scattering by Particles: Computational Methods (World Scientific, Singapore, 1990), Chap. 3.

[12] A. Doicu, T. Wriedt, and Y. A. Eremin, Light Scattering by Systems of Particles: Null-Field Method with Discrete SourcesTheory and Programs (Springer, Berlin, 2006), Chap. 2.

[13] P. Debye, Phys. Zeit. 9, 775 (1908); which was translated into English and reprinted in "Selected Papers on Geometrical Aspects of Scattering," edited by P. L. Marston, SPIE Milestone Series vol. MS89, 198 (1994).
[14] B. Van der Pol and H. Bremmer, Philos. Mag. 24, 141, 825 (1937).

[15] H. M. Nussenzveig, J. Math. Phys. 10, 82 (1969).

[16] J. A. Lock, J. Opt. Soc. Am. A 5, 2032 (1988).

[17] J. A. Lock, J. M. Jamison, and C.-Y. Lin, Appl. Opt. 33, 4677 (1994).

[18] J. A. Lock, Appl. Opt. 44, 5594 (2005).

[19] J. A. Lock, J. Opt. Soc. Am. A 25, 2980 (2008).

[20] J. A. Lock, J. Opt. Soc. Am. A 18, 3085 (2001).

[21] J. A. Lock and C. L. Adler, J. Opt. Soc. Am. A 14, 1316 (1997).

[22] G. Gouesbet, Part. Part. Syst. Charact. 20, 382 (2003).

[23] Z. Wu and H. Li, Chin. Phys. Lett. 25, 1672 (2008).

[24] R. Li, X. Han, L. Shi, K. F. Ren, and H. Jiang, Appl. Opt. 46, 4804 (2007).

[25] F. Xu, J. A. Lock, and C. Tropea, J. Opt. Soc. Am. A 27, 671 (2010).

[26] M. Kerker, The Scattering of Light and Other Electromagnetic Radiation (Academic Press, New York, 1969), Chap 6.

[27] P. M. Morse and H. Feshbach, Methods of Theoretical Physic (McGraw-Hill, New York, 1953), Chap 5.

[28] K. N. Liou, An Introduction to Atmospheric Radiation, 2nd ed. (Academic Press, San Diego, 2002).

[29] R. J. Adrian, D. F. G. Durao, F. Durst, M. V. Heitor, M. Maeda, and J. H. Whitelaw, Laser Techniques Applied to Fluid Mechanics (Springer, Berlin, 2000). 
[30] G. Gouesbet, B. Maheu, and G. Gréhan, J. Opt. Soc. Am. A 5, 1427 (1988).

[31] Ref. [10], Appendix C.

[32] B. Maheu, G. Gouesbet, and G. Gréhan, J. Opt. (Paris) 19, 59 (1988).

[33] G. Gouesbet, C. Letellier, K. F. Ren, and G. Gréhan, Appl. Opt. 35, 1537 (1996).

[34] G. Gouesbet, G. Gréhan, and B. Maheu, Appl. Opt. 27, 4874 (1988).

[35] G. Gouesbet, G. Gréhan, and B. Maheu, J. Opt. (Paris) 19, 35 (1988).

[36] G. Gouesbet, J. Opt. Soc. Am. A 16, 1641 (1999).

[37] K. F. Ren, G. Gouesbet, and G. Gréhan, Appl. Opt. 37, 4218 (1998).

[38] A. Doicu and T. Wriedt, Appl. Opt. 36, 2971 (1997).

[39] H. Zhang and Y. Han, J. Opt. Soc. Am. B 25, 255 (2008).
[40] H. C. van de Hulst, Light Scattering by Small Particles (Dover, New York, 1981), Chap. 9.

[41] C. F. Bohren and D. R. Huffman, Absorption and Scattering of Light by Small Particles (Wiley, New York, 1983), Chap. 4.

[42] P. Barber and C. Yeh, Appl. Opt. 14, 2864 (1975).

[43] Ref. [25], Chaps. 7, 11, and 13.

[44] G. Arfken, Mathematical Methods for Physicists, 3rd ed., (Academic Press, New York, 1985), Chap. 11. Note that Eqs. (30) and (31) are obtained from Exercises 11.7.6 and 11.7.7, respectively.

[45] A. Doicu and T. Wriedt, J. Quant. Spectrosc. Radiat. Transfer 111, 466 (2010).

[46] J. A. Lock and F. Xu, Appl. Opt. 49, 1288 (2010).

[47] M. Born and E. Wolf, Principles of Optics, 6th corrected ed. (Pergamon, New York, 1980), pp. 398-399.

[48] J. A. Lock, Appl. Opt. 35, 500 (1996).

[49] E. A. Hovenac and J. A. Lock, J. Opt. Soc. Am. A 9, 781 (1992). 